\title{
IRREDUCIBLE REPRESENTATIONS OF THE EXCEPTIONAL CHENG-KAC SUPERALGEBRA
}

\author{
CONSUELO MARTÍNEZ AND EFIM ZELMANOV \\ To the memory of our dear friend Hyo Chul Myung
}

\begin{abstract}
We classify all conformal irreducible modules of finite type over
\end{abstract} the Cheng-Kac superalgebra $\mathrm{CK}_{6}$.

\section{INTRODUCTION}

The study of Lie conformal superalgebras and their representations was initiated by V. Kac ( $[9]$ ) in view of their connection to the free fields realizations in conformal field theory. A complete classification of simple Lie conformal superalgebras of finite type was achieved in [6]. The list consists of current Lie superalgebras, $\operatorname{Cur}(\mathcal{G})$, where $\mathcal{G}$ is a simple finite dimensional Lie superalgebra, four series of Lie conformal superalgebras of Cartan type and the exceptional Lie conformal superalgebra $C K_{6}$.

For the classification of representations of finite type of current Lie superalgebras and Lie superalgebras of Cartan type see [3, [1, [2, 4].

In this paper we classify all conformal irreducible modules of finite type over the superalgebra $C K_{6}$. We use this classification and the results of [13] to classify conformal irreducible Jordan bimodules of finite type over the Jordan superalgebra $J C K(6)$.

A different approach to this classification is outlined in a series of papers by C. Boyallian, V.G. Kac and J.I. Liberati (see, for example, [2]). They use the methods that worked well for other classes of conformal superalgebras: a description of extremal vectors and degenerate modules, whereas our approach is specific for $C K_{6}$. It is based on the explicit matrix realization of $C K_{6}$ over the Weyl algebra (see [10]) and its rich weight structure. It would be interesting to establish a direct link between the two descriptions.

\section{BASIC DEFINITIONS}

Let $A$ be an arbitrary (not necessarily associative) algebra over C. By a formal distribution

$$
a(z)=\sum_{i \in \mathbf{Z}} a(i) z^{-i-1} \in A[[z]]
$$

we mean a power series over $A$, which is infinite in both directions.

Two formal distributions $a(z), b(z)$ are said to be mutually local if there exists an integer $N=N(a, b) \geq 0$ such that $a(z) b(w)(z-w)^{N}=b(w) a(z)(z-w)^{N}=0$.

Received by the editors August 3, 2012 and, in revised form, December 7, 2012.

2010 Mathematics Subject Classification. Primary 17C70; Secondary 17C55, 17B10, 17B60.

The first author was partially supported by MTM 2010-67884-C04-01.

The second author was partially supported by the NSF. 
We will consider a countable family of operations:

$$
a(z) \circ_{n} b(z)=\operatorname{Res}_{w} a(w) b(z)(w-z)^{n}, n \geq 0, n \in \mathbf{Z} .
$$

Here $\operatorname{Res}_{w}$ means the coefficient at $w^{-1}$.

If $a(z), b(z)$ are mutually local, then only finitely many products $a \circ_{n} b$ may be different from zero.

Definition 1.1. A vector space $C \subseteq A\left[\left[z^{-1}, z\right]\right]$ is called a conformal algebra of formal distributions if $\partial C \subseteq C, \partial=\frac{d}{d z}, C \circ_{n} C \subseteq C$ for an arbitrary $n \geq 0$ and every two elements from $C$ are mutually local.

By Dong's Lemma (see [9]), if $A$ is an associative or Lie algebra, then for an arbitrary collection $C$ of pairwise mutually local distributions the closure of $C$ with respect to the action of $\partial$ and to all operations $\circ_{n}, n \geq 0$, is a conformal algebra of formal distributions.

Example 1.2. (1) Let $\mathcal{G}$ be an arbitrary algebra and let $A=\mathcal{G}\left[t, t^{-1}\right]$ be the algebra of Laurent polynomials over $\mathcal{G}$. For an arbitrary element $a \in \mathcal{G}$ let $\tilde{a}=$ $\sum_{i \in \mathbf{Z}}\left(a t^{i}\right) z^{-i-1} \in A\left[\left[z^{-1}, z\right]\right]$.

Any two formal distributions $\tilde{a}, \tilde{b}$ are mutually local.

(2) Let $\mathcal{V}$ ir $=\operatorname{Der} \mathbf{C}\left[t^{-1}, t\right]$ be the (centerless) Virasoro algebra. The formal distribution

$$
L=\sum_{i \in \mathbf{Z}} t^{i+1} \frac{d}{d t} z^{-i-2} \in \mathcal{V} i r\left[\left[z^{-1}, z\right]\right]
$$

is mutually local with itself.

(3) Let $W=\left\langle t^{-1}, t, \frac{d}{d t}\right\rangle$ be the (associative) Weyl algebra of differential operators on $C\left[t^{-1}, t\right]$. Let $J_{k}=\sum_{i \in Z} t^{i}\left(\frac{d}{d t}\right)^{k} z^{-i-1}, k \geq 0$. Any two formal distributions $J_{k}$, $J_{l}$ are mutually local.

In all three of these cases we can talk about the conformal algebras $C u r(\mathcal{G}), \mathcal{V} i r$, $W$ respectively, generated by them.

Now we are ready to introduce an abstract definition of a conformal algebra.

Let $C$ be a module over a polynomial algebra $\mathbf{C}[\partial]$, which is equipped with countably many binary bilinear operations $C \circ_{n} C \rightarrow C, n \geq 0$.

Definition 1.3. We say that $\left(C, \partial, \circ_{n}\right)$ is an abstract conformal algebra if for arbitrary elements $a, b \in C$ and arbitrary $n \geq 0$, we have:

1) $\partial\left(a \circ_{n} b\right)=\partial a \circ_{n} b+a \circ_{n} \partial b$.

2) $\partial a \circ_{n} b=-n a \circ_{n-1} b$; for $n=0$ the condition turns into $\partial a \circ_{0} b=0$.

3) (Locality) There exists an integer $N=N(a, b) \geq 0$ such that for an arbitrary $n \geq N$ we have $a \circ_{n} b=0$.

Every conformal algebra of formal distributions is an abstract conformal algebra. The converse is also true: every conformal algebra can be realized as an algebra of formal distributions over some algebra of coefficients. Moreover, among these algebras of coefficients there is a universal one $\operatorname{Coeff}(C)$.

Definition 1.4. We say that a conformal algebra $C$ is a Lie (resp. associative, Jordan) algebra iff $\operatorname{Coeff}(C)$ is a Lie (associative, Jordan) algebra.

Now let $C$ be a Lie conformal algebra and let $M$ be another $\mathbf{C}[\partial]$-module. Suppose that we have a family of bilinear maps $C \circ_{n} M \subseteq M, n \geq 0$. 
Definition 1.5. We say that $M$ is a conformal $C$-module if the null split extension $C+M$ is a Lie conformal algebra.

As above, $M$ can be realized as a space of formal distributions over $\operatorname{Coeff}(M)$, where $\operatorname{Coeff}(M)$ is a universal (with this property) Lie module over $\operatorname{Coeff}(C)$.

Important Remark. If there is a natural (and standard) way to arrange elements of a (super)algebra $L$ in formal distributions, then we will talk about $L$ and modules over $L$ even if we have in mind their conformal counterparts.

\section{The Cheng-KaC superalgebra}

The exceptional conformal superalgebra $C K_{6}$ was introduced in [5] and in [7]. In [10] we constructed, for an arbitrary associative commutative superalgebra $R$ with an even derivation $d: R \rightarrow R$, a superalgebra $C K(R, d)$ so that $C K_{6} \simeq$ $C K\left(\mathbf{C}\left[t^{-1}, t\right], \frac{d}{d t}\right)$.

Let's recall the construction of $C K(R, d)$ from [10].

Consider the associative Weyl algebra $W=\sum_{i \geq 0} R d^{i}$, where the variable $d$ does not commute with a coefficient $a \in R$, but $d a=a d+d(a)$. We will realize $C K(R, d)$ as a superalgebra of $8 \times 8$ matrices over $W$.

The simple finite dimensional Lie superalgebra $P(n-1)$ is the superalgebra of $2 n \times 2 n$ matrices of the type $\left(\begin{array}{cc}a & k \\ h & -a^{t}\end{array}\right)$, where $a, h, k$ are $n \times n$-matrices over $\mathbf{C}$, $\operatorname{tr}(a)=0, k^{t}=-k, h^{t}=h$. The superalgebras $P(n), n \neq 3$, are centrally closed. However, $P(3)$ has a nontrivial central cover $\hat{P}(3)$. Its existence comes from the fact that the Lie algebra $K_{4}(\mathbf{C})$ of skew-symmetric $4 \times 4$ matrices is a direct sum of two ideals $K_{4}(\mathbf{C})=s l_{2}(\mathbf{C}) \oplus s l_{2}(\mathbf{C})$. For an arbitrary element $k \in K_{4}(\mathbf{C})$ we consider its decomposition $k=k^{\prime}+k^{\prime \prime}$ and let $\varphi(k)=k^{\prime}-k^{\prime \prime}$. The universal central cover $\hat{P}(3)$ of $P(3)$ can be realized as a superalgebra of $8 \times 8$ matrices over the polynomial algebra $\mathbf{C}[d]$ of the type

$$
\left(\begin{array}{cc}
a & k \\
\varphi(k) d+h & -a^{t}
\end{array}\right)+\alpha d I_{8}
$$

where $a, k, h$ are $4 \times 4$ matrices over $\mathbf{C}, \operatorname{tr}(a)=0, k=-k^{t}, h=h^{t}, \alpha \in \mathbf{C}$ and $I_{8}$ is the identity matrix.

The superalgebra $C K(R, d)$ is a subsuperalgebra of $8 \times 8$ matrices over $W$ generated by $\hat{P}(3)$ and by all matrices $\left(\begin{array}{cc}e_{i j}(a) & 0 \\ 0 & -e_{j i}(a)\end{array}\right)$ where $a \in R, 1 \leq i \neq j \leq 4$.

The Cartan subalgebra $H$ of $C K(R, d)$ consists of diagonal matrices

$$
H=\left\{h=\operatorname{diag}\left(a_{1}, \ldots, a_{4},-a_{1}, \ldots,-a_{4}\right), a_{i} \in \mathbf{C}, \sum_{i=1}^{4} a_{i}=0\right\},
$$

and the even and the odd roots of $C K(R, d)$ with respect to the action of $H$ are

$$
\begin{gathered}
\Delta_{\overline{0}}=\left\{w_{i}-w_{j} \mid 1 \leq i \neq j \leq 4\right\}, \\
\Delta_{\overline{1}}=\left\{w_{i}+w_{j}, 1 \leq i \neq j \leq 4,-w_{i}-w_{j}, 1 \leq i, j \leq 4\right\} .
\end{gathered}
$$

Let us denote $\Delta=\Delta_{\overline{0}} \cup \Delta_{\overline{1}}$. Notice that $w_{i}(h)=a_{i}, 1 \leq i \leq 4$. 
Thus, the superalgebra $C K(R, d)$ is graded by the abelian group

$$
\sum_{i=1}^{4} \mathbf{Z} w_{i} / \mathbf{Z}\left(w_{1}+w_{2}+w_{3}+w_{4}\right)
$$

$C K(R, d)=\sum_{\alpha \in \Delta \cup\{0\}} C K(R, d)_{\alpha}$.

Let us fix the notation for the following weight elements:

$$
\begin{gathered}
e_{w_{i}-w_{j}}=\left(\begin{array}{cc}
e_{i j} & 0 \\
0 & -e_{j i}
\end{array}\right), e_{w_{i}-w_{j}}(a)=\left(\begin{array}{cc}
e_{i j}(a) & 0 \\
0 & -e_{j i}(a)
\end{array}\right), \\
h_{w_{i}-w_{j}}(a)=\left(\begin{array}{cc}
e_{i i}(a)-e_{j j}(a) & 0 \\
0 & e_{j j}(a)-e_{i i}(a)
\end{array}\right), q_{-w_{i}-w_{j}}=\left(\begin{array}{cc}
0 & 0 \\
e_{i j}+e_{j i} & 0
\end{array}\right), \\
q_{-w_{i}-w_{j}}(a)=\left(\begin{array}{cc}
0 & 0 \\
e_{i j}(a)+e_{j i}(a) & 0
\end{array}\right), q_{w_{i}+w_{j}}=\left(\begin{array}{cc}
0 & e_{i j}-e_{j i} \\
\varphi\left(e_{i j}-e_{j i}\right) d & 0
\end{array}\right),
\end{gathered}
$$

$a \in R$.

In [12] it was shown that $C K(R, d)_{w_{i}-w_{j}}=e_{w_{i}-w_{j}}(R), 1 \leq i \neq j \leq 4$; $C K(R, d)_{-2 w_{i}}=q_{-2 w_{i}}(R) ; C K(R, d)_{w_{i}+w_{j}}=\left[q_{w_{i}+w_{k}}, e_{w_{j}-w_{k}}(R)\right]+q_{-w_{k}-w_{l}}(R)$, where $\{i, j, k, l\}=\{1,2,3,4\}$.

For an arbitrary element $a \in R$ consider the element

$$
\begin{gathered}
{\left[\left[e_{w_{4}-w_{1}}(a), q_{w_{3}+w_{1}}\right], q_{w_{2}+w_{1}}\right]} \\
=\left(\begin{array}{cc}
e_{11}(d a)+e_{22}(a d)+e_{33}(a d)+e_{44}(a d) & 0 \\
0 & e_{11}(a d)+e_{22}(d a)+e_{33}(d a)+e_{44}(d a)
\end{array}\right) \\
=I_{8}(a d)-\left(\begin{array}{cc}
e_{11}\left(a^{\prime}\right) & 0 \\
0 & -e_{11}\left(a^{\prime}\right)+I_{4}\left(a^{\prime}\right)
\end{array}\right), a^{\prime}=[a, d]=d(a) .
\end{gathered}
$$

We will denote the element on the right hand side as $\operatorname{Vir}(a)$. The mapping $a d \rightarrow \operatorname{Vir}(a)$ from $R d \rightarrow \mathcal{V} \operatorname{ir}(R)$ is an isomorphism of Lie algebras.

It was shown in 12 that $C K(R, d)_{0}=H \otimes R+\mathcal{V} \operatorname{ir}(R)$.

Consider the functional

$$
f: \sum_{i=1}^{4} \mathbf{Z} w_{i} / \mathbf{Z}\left(\sum_{i=1}^{4} w_{i}\right) \rightarrow \mathbf{Z}
$$

given by $f\left(w_{1}\right)=5, f\left(w_{2}\right)=-3, f\left(w_{3}\right)=2, f\left(w_{4}\right)=-4$.

Notice that $f\left( \pm w_{i} \pm w_{j}\right) \neq 0$, unless $\pm w_{i} \pm w_{j}=0$.

From now on we will denote $L=C K_{6}=C K\left(\mathbf{C}\left[t^{-1}, t\right], \frac{d}{d t}\right)$.

Note that $L_{0}=H \otimes \mathbf{C}\left[t^{-1}, t\right] \ngtr \mathcal{V} i r(R) \leq C u r\left(s l_{4}\right) \rtimes \mathcal{V} i r(R) \leq L$.

The algebra $L$ has a triangular decomposition

$$
L=L_{-}+L_{0}+L_{+}, L_{-}=\sum_{f(\alpha)<0} L_{\alpha}, L_{+}=\sum_{f(\alpha)>0} L_{\alpha} .
$$

Let $M$ be a conformal module of finite type over the Lie conformal algebra $C K_{6}$. Then the subalgebra $s l_{4} \subseteq L$ acts on $M$ and the action of $s l_{4}$ commutes with the action of the polynomial algebra $\mathbf{C}[\partial]$. Hence $M$ decomposes into a finite direct sum of eigenspaces with respect to the action of $H$,

$$
M=\sum_{\gamma \in H^{*}} M_{\gamma}
$$


If $M$ is irreducible, then there exists a unique highest weight $\lambda \in H^{*}$ such that $M_{\lambda} \neq(0)$ and $L_{+}{ }^{\circ}{ }_{n} M_{\lambda}=(0)$ for all $n \geq 0 ; M_{\lambda}$ is an irreducible conformal module over $L_{0}$.

We have mentioned above that $L_{0} \subset C u r\left(s l_{4}\right)+\mathcal{V}$ ir $\subset L$.

Let $M^{\prime}$ be the $\operatorname{Cur}\left(s l_{4}\right)+\mathcal{V} i r$-module generated by $M_{\lambda}$. Let $M^{\prime \prime}$ be the largest submodule of $M^{\prime}$ such that $M^{\prime \prime} \cap M_{\lambda}=0$. Then $M^{\prime} / M^{\prime \prime}$ is an irreducible Cur $\left(s l_{4}\right)+$ Vir-module and $\left(M^{\prime} / M^{\prime \prime}\right)_{\lambda}=M_{\lambda}$. Let $V_{\lambda}=\operatorname{Coeff}(M)$ be an $L$-module.

From the description of irreducible modules of finite type over $\operatorname{Cur}\left(s l_{4}\right)>\triangleleft \mathcal{V} \operatorname{ir}(R)$ (see [4]), it follows that the module $V_{\lambda}$ can be identified with $\mathbf{C}\left[t^{-1}, t\right]$, say $V_{\lambda}=\overline{\mathbf{C}\left[t^{-1}, t\right]}$. For arbitrary elements $a, b \in \mathbf{C}\left[t^{-1}, t\right], h \in H$ we have $(h \otimes a) \bar{b}=\langle\lambda, h\rangle \overline{a b}$. Moreover, there exist scalars $\alpha, \beta \in \mathbf{C}$ such that for arbitrary $a, b \in \mathbf{C}\left[t^{-1}, t\right]$ we have

$$
\operatorname{Vir}(a) \bar{b}=\overline{-a b^{\prime}+\beta a^{\prime} b+\alpha a b} .
$$

Denote this $L_{0}$-module as $V(\lambda, \beta, \alpha)$. It is well known that, for an arbitrary $\lambda \in H^{*}$, given an irreducible $L_{0}$-module $W$ such that the elements $h \in H$ act on $W$ as scalar multiplications $\langle\lambda, h\rangle$, there exists a unique $L$-module with the highest weight $\lambda$ under the action of $H$, whose $\lambda$-space is isomorphic to $W$ as an $L_{0^{-}}$ module. If we consider the irreducible $L_{0}$-module $V(\lambda, \beta, \alpha)$, then the corresponding irreducible $L$-module will be denoted as $\operatorname{Irr}(\lambda, \beta, \alpha)$.

It follows from above that every irreducible conformal module over $C K_{6}$ is isomorphic to $\operatorname{Irr}(\lambda, \beta, \alpha)$ for some $\lambda \in H^{*}, \beta, \alpha \in \mathbf{C}$. This gives rise to the question:

For which parameters $\lambda \in H^{*}, \beta, \alpha \in \mathbf{C}$ is the irreducible conformal module $\operatorname{Irr}(\lambda, \Delta, \alpha)$ of finite type?

Let $\lambda$ be an integral dominant weight, that is, $\left\langle\lambda, h_{w_{1}-w_{3}}\right\rangle,\left\langle\lambda, h_{w_{3}-w_{2}}\right\rangle$, and $\left\langle\lambda, h_{w_{2}-w_{4}}\right\rangle$ all lie in $\mathbf{Z}_{\geq 0}$.

Theorem 2.1. The conformal module $\operatorname{Irr}(\lambda, \beta, \alpha)$ is of finite type if and only if

(1) $\left\langle\lambda, h_{w_{1}-w_{3}}\right\rangle \geq 2 ; \beta, \alpha \in \mathbf{C}$, or

(2) $\left\langle\lambda, h_{w_{1}-w_{3}}\right\rangle=1 ;\left\langle\lambda, h_{w_{2}-w_{3}}\right\rangle=0, \beta=-1, \alpha \in \mathbf{C}$.

These modules exhaust all conformal irreducible $C K_{6}$-modules of finite type.

Since $V(\lambda, \beta, \alpha)$ are known to be conformal modules of finite type over $L_{0}$ (see [4]), we can easily conclude that $\operatorname{Irr}(\lambda, \beta, \alpha)$ is of finite type if and only if it has finitely many weights with respect to the action of $H$. At this point we can forget about conformal modules and address the question:

For which $\lambda \in H^{*}, \beta, \alpha \in \mathbf{C}$ does the $L$-module $\operatorname{Irr}(\lambda, \beta, \alpha)$ have finitely many weights?

Lemma 2.2. Let $\alpha=w_{i}-w_{j}$ or $-w_{i}-w_{j}$. For an element $a \in R$, let $X_{\alpha}(a)=$ $e_{w_{i}-w_{j}}(a)$ or $q_{-w_{i}-w_{j}}(a)$ be defined as above. Suppose that $\alpha<0$ and for any decomposition $-\alpha=\alpha_{1}+\cdots+\alpha_{r}$ into a sum of positive roots, for any elements $x_{i} \in L_{\alpha_{i}}, 1 \leq i \leq r$, there exist an element $h \in H$ and an element $b \in R$ such that $\left[x_{1},\left[x_{2}, \ldots\left[x_{r}, X_{\alpha}(a)\right] \cdots\right]=h \otimes a b\right.$ for an arbitrary $a \in R$. Then for an arbitrary element $v_{\lambda} \in V_{\lambda}$ we have $X_{\alpha}(a) v_{\lambda}=X_{\alpha}(1)(a v)_{\lambda}$.

Proof. It is sufficient to show that

$$
U\left(L_{+}\right)\left(X_{\alpha}(a) v_{\lambda}-X_{\alpha}(1)(a v)_{\lambda}\right) \cap V_{\lambda}=(0) .
$$

Otherwise there exists a decomposition $-\alpha=\alpha_{1}+\cdots+\alpha_{r}, \alpha_{i}>0$ and elements $x_{i} \in L_{\alpha_{i}}$ such that $x_{1} \cdots x_{r}\left(X_{\alpha}(a) v_{\alpha}-X_{\alpha}(1)(a v)_{\lambda}\right) \neq 0$. 
But

$$
\begin{gathered}
x_{1} \cdots x_{r} X_{\alpha}(a) v_{\lambda}=\left[x_{1},\left[x_{2}, \ldots,\left[x_{r}, X_{\alpha}(a)\right] \ldots\right]\right] v_{\lambda}=(h \otimes a b) v_{\lambda} \\
=h(a b v)_{\lambda}=\left[x_{1},\left[x_{2}, \ldots,\left[x_{r}, X_{\alpha}(1)\right] \ldots\right]\right](a v)_{\lambda}=x_{1} \ldots x_{r} X_{\alpha}(1)(a v)_{\lambda},
\end{gathered}
$$

a contradiction. The lemma is proved.

Lemma 2.3. The negative roots $w_{2}-w_{3}, w_{4}-w_{3},-w_{1}-w_{2},-w_{1}-w_{3},-w_{1}-w_{4}$, $w_{4}-w_{2}$ satisfy the assumptions of Lemma 2.2.

Proof. We list all possible decompositions. The roots $w_{3}-w_{2}, w_{1}+w_{4}$ and $w_{2}-w_{4}$ do not have nontrivial decompositions. Then, $w_{1}+w_{2}=\left(w_{1}+w_{4}\right)+\left(w_{2}-w_{4}\right)$, $w_{1}+w_{3}=\left(w_{1}+w_{2}\right)+\left(w_{3}-w_{2}\right)=\left(w_{1}+w_{4}\right)+\left(w_{2}-w_{4}\right)+\left(w_{3}-w_{2}\right)=$ $\left(w_{3}-w_{4}\right)+\left(w_{4}+w_{1}\right) ; w_{3}-w_{4}=\left(w_{2}-w_{4}\right)+\left(w_{3}-w_{2}\right)$.

The condition of Lemma 2.2 is checked by a straightforward computation in the superalgebra $L$. The lemma is proved.

Lemma 2.4. For arbitrary elements $a, b \in R$ we have

$$
\left[q_{w_{1}+w_{4}}, e_{w_{2}-w_{4}}(a)\right]\left[q_{w_{1}+w_{4}}, e_{w_{3}-w_{4}}(b)\right] q_{-w_{1}-w_{2}} q_{-w_{1}-w_{3}} v_{\lambda}=\gamma(a b v)_{\lambda}
$$

where $\gamma=\left\langle\lambda, h_{w_{1}-w_{2}}\right\rangle\left(1-\left\langle\lambda, h_{w_{1}-w_{3}}\right\rangle\right)$.

Proof. We have $\left[q_{w_{1}+w_{4}}, e_{w_{3}-w_{4}}(b)\right] q_{-w_{1}-w_{2}} q_{-w_{1}-w_{3}} v_{\lambda}=(I)-(I I)$, where

$$
\begin{gathered}
(I)=q_{w_{1}+w_{4}} e_{w_{3}-w_{4}}(b) q_{-w_{1}-w_{2}} q_{-w_{1}-w_{3}} v_{\lambda} \\
=q_{w_{1}+w_{4}} q_{-w_{1}-w_{2}} e_{w_{3}-w_{4}}(b) q_{-w_{1}-w_{3}} v_{\lambda} \\
=q_{w_{1}+w_{4}} q_{-w_{1}-w_{2}}\left[e_{w_{3}-w_{4}}(b), q_{-w_{1}-w_{3}}\right] v_{\lambda}+q_{w_{1}+w_{4}} q_{-w_{1}-w_{2}} q_{-w_{1}-w_{3}} e_{w_{3}-w_{4}}(b) v_{\lambda} \\
=-q_{w_{1}+w_{4}} q_{-w_{1}-w_{2}} q_{-w_{1}-w_{4}}(b) v_{\lambda}=-q_{w_{1}+w_{4}} q_{-w_{1}-w_{2}} q_{-w_{1}-w_{4}}(b v)_{\lambda},
\end{gathered}
$$

by Lemma 3.1. Then

$$
\begin{gathered}
(I I)=e_{w_{3}-w_{4}}(b) q_{w_{1}+w_{4}} q_{-w_{1}-w_{2}} q_{-w_{1}-w_{3}} v_{\lambda} \\
=-e_{w_{3}-w_{4}}(b) e_{w_{4}-w_{2}} q_{-w_{1}-w_{3}} v_{\lambda}-e_{w_{3}-w_{4}}(b) q_{-w_{1}-w_{2}} q_{w_{1}+w_{4}} q_{-w_{1}-w_{3}} v_{\lambda} \\
=-e_{w_{3}-w_{4}}(b) q_{-w_{1}-w_{3}} e_{w_{4}-w_{2}} v_{\lambda}-q_{-w_{1}-w_{2}} e_{w_{3}-w_{4}}(b) q_{w_{1}+w_{4}} q_{-w_{1}-w_{3}} v_{\lambda} .
\end{gathered}
$$

Now

$$
=\left[\begin{array}{c}
e_{w_{3}-w_{4}}(b) q_{-w_{1}-w_{3}} e_{w_{4}-w_{2}} v_{\lambda} \\
=\left[e_{w_{3}-w_{4}}(b), q_{-w_{1}-w_{3}}\right] e_{w_{4}-w_{2}} v_{\lambda}+q_{-w_{1}-w_{3}} e_{w_{3}-w_{4}}(b) e_{w_{4}-w_{2}} v_{\lambda} .
\end{array}\right.
$$

The second summand is 0 since $f\left(w_{3}-w_{4}\right)=6$, whereas $f\left(w_{4}-w_{2}\right)=-1$.

The first summand is

$$
\begin{gathered}
-q_{-w_{1}-w_{4}}(b) e_{w_{4}-w_{2}} v_{\lambda} \\
=-\left[q_{-w_{1}-w_{4}} e_{w_{4}-w_{2}}\right] v_{\lambda}-e_{w_{4}-w_{2}} q_{-w_{1}-w_{4}}(b) v_{\lambda} \\
=-q_{-w_{1}-w_{2}}(b) v_{\lambda}-e_{w_{4}-w_{2}} q_{-w_{1}-w_{4}}(b) v_{\lambda} \\
=-q_{-w_{1}-w_{2}}(b v)_{\lambda}-e_{w_{4}-w_{2}} q_{-w_{1}-w_{4}}\left(b v_{\lambda}\right),
\end{gathered}
$$

by Lemma 2.3.

As for the other summand of (II),

$$
\begin{gathered}
q_{-w_{1}-w_{2}} e_{w_{3}-w_{4}}(b) q_{w_{1}+w_{4}} q_{-w_{1}-w_{3}} v_{\lambda} \\
=q_{-w_{1}-w_{2}}\left[e_{w_{3}-w_{4}}(b),\left[q_{w_{1}+w_{4}}, q_{-w_{1}-w_{3}}\right]\right] v_{\lambda} \\
=q_{-w_{1}-w_{2}} h_{w_{3}-w_{4}}(b) v_{\lambda}=q_{-w_{1}-w_{2}} h_{w_{3}-w_{4}}(b v)_{\lambda} .
\end{gathered}
$$

We proved that

$$
\left[q_{w_{1}+w_{4}}, e_{w_{3}-w_{4}}(b)\right] q_{-w_{1}-w_{2}} q_{-w_{1}-w_{3}} v_{\lambda}=P(b v)_{\lambda},
$$


where $P$ is an operator that does not involve $b$. Choosing $b=1$ we get

$$
\begin{gathered}
P=\operatorname{ad}\left(\left[q_{w_{1}+w_{4}}, e_{w_{3}-w_{4}}\right]\right) \operatorname{ad}\left(q_{-w_{1}-w_{2}}\right) \operatorname{ad}\left(q_{-w_{1}-w_{3}}\right) \\
=\operatorname{ad}\left(q_{w_{3}+w_{1}}\right) \operatorname{ad}\left(q_{-w_{1}-w_{2}}\right) \operatorname{ad}\left(q_{-w_{1}-w_{3}}\right) .
\end{gathered}
$$

Now we have to consider the element

$$
\left[q_{w_{1}+w_{4}}, e_{w_{2}-w_{4}}(a)\right] q_{w_{3}+w_{1}} q_{-w_{1}-w_{2}} q_{-w_{1}-w_{3}}(b v)_{\lambda} .
$$

Remark that $\left[\left[q_{w_{1}+w_{4}}, e_{w_{2}-w_{4}}(a)\right], q_{w_{3}+w_{1}}\right] \in e_{w_{1}-w_{4}}(R)$ and

$$
e_{w_{1}-w_{4}}(R) q_{-w_{1}-w_{2}} q_{-w_{1}-w_{3}}(b v)_{\lambda}=\left[\left[e_{w_{1}-w_{4}}(R), q_{-w_{1}-w_{2}}\right], q_{-w_{1}-w_{3}}\right](b v)_{\lambda}=(0) \text {. }
$$

Hence our expression becomes

$$
-q_{w_{1}+w_{3}}\left[q_{w_{1}+w_{4}}, e_{w_{2}-w_{4}}(a)\right] q_{-w_{1}-w_{2}} q_{-w_{1}-w_{3}}(b v)_{\lambda} .
$$

Denote $X=\left[q_{w_{1}+w_{4}}, e_{w_{2}-w_{4}}(a)\right], Y=q_{-w_{1}-w_{2}}, Z=q_{-w_{1}-w_{3}}$. Then $X Y Z=$ $[[X, Y], Z]-Y[X, Z]+Y Z X+Z[X, Y],[X, Y]=h_{w_{2}-w_{1}}(a),[X, Z]=e_{w_{2}-w_{3}}(a)$, $[[X, Y], Z]=q_{-w_{1}-w_{3}}(a)$. By Lemma 2.3,

$$
h_{w_{2}-w_{1}}(a)(b v)_{\lambda}=h_{w_{2}-w_{1}}(a b v)_{\lambda}, e_{w_{2}-w_{3}}(a)(b v)_{\lambda}=e_{w_{2}-w_{3}}(a b v)_{\lambda}
$$

and

$$
q_{-w_{1}-w_{3}}(a)(b v)_{\lambda}=q_{-w_{2}-w_{3}}(a b v)_{\lambda} .
$$

As we did above, we can conclude that

$$
\left[q_{w_{1}+w_{4}}, e_{w_{2}-w_{4}}(a)\right]\left[q_{w_{1}+w_{4}}, e_{w_{3}-w_{4}}(b)\right] q_{-w_{1}-w_{2}} q_{-w_{1}-w_{3}} v_{\lambda}=\tilde{P}(a b v)_{\lambda},
$$

where $\tilde{P}$ is an operator that does not involve $a$ or $b$. Choosing $a=b=1$ we get

$$
\tilde{P}=\operatorname{ad}\left(q_{w_{2}+w_{1}}\right) \operatorname{ad}\left(q_{w_{3}+w_{1}}\right) \operatorname{ad}\left(q_{-w_{1}-w_{2}}\right) \operatorname{ad}\left(q_{-w_{1}-w_{3}}\right) .
$$

Now

$$
\begin{gathered}
\tilde{P}(a b v)_{\lambda}=q_{w_{2}+w_{1}}\left[q_{w_{3}+w_{1}}, q_{-w_{1}-w_{2}}\right] q_{-w_{1}-w_{3}}(a b v)_{\lambda} \\
-q_{w_{2}+w_{1}} q_{-w_{1}-w_{2}} q_{w_{3}+w_{1}} q_{-w_{1}-w_{3}}(a b v)_{\lambda} \\
=\left[q_{w_{2}+w_{1}},\left[\left[q_{w_{3}+w_{1}}, q_{-w_{1}-w_{2}}\right], q_{-w_{1}-w_{3}}\right]\right](a b v)_{\lambda} \\
-\left[\left[q_{w_{2}+w_{1}}, q_{-w_{1}-w_{2}}\right],\left[q_{w_{3}+w_{1}}, q_{-w_{1}-w_{3}}\right]\right](a b v)_{\lambda} \\
=-h_{w_{2}-w_{1}}(a b v)_{\lambda}-h_{w_{2}-w_{1}} h_{w_{3}-w_{1}}(a b v)_{\lambda}=\gamma(a b v)_{\lambda},
\end{gathered}
$$

$\gamma=\left\langle\lambda, h_{w_{1}-w_{2}}\right\rangle\left(1-\left\langle\lambda, h_{w_{1}-w_{3}}\right\rangle\right)$. The lemma is proved.

Remark. In what follows $\left[x_{1}, \ldots, x_{n}\right]$ denotes the left-normed commutator $\left[\left[\ldots\left[x_{1}, x_{2}\right], x_{3}\right], \ldots, x_{n}\right]$.

Lemma 2.5. $\left[\left[e_{w_{4}-w_{1}}(a),\left[q_{w_{1}+w_{4}}, e_{w_{3}-w_{4}}(c)\right]\right],\left[q_{w_{1}+w_{4}}, e_{w_{2}-w_{4}}(b)\right]\right]=h_{w_{1}-w_{4}}\left(a b^{\prime} c\right)$ $-\operatorname{Vir}(a b c)$.

Proof. Since $\left[e_{w_{4}-w_{1}}(a), q_{w_{1}+w_{4}}\right]=0$ the left hand side is equal to

$$
\begin{gathered}
{\left[e_{w_{4}-w_{1}}(a), e_{w_{3}-w_{4}}(c), q_{w_{1}+w_{4}}, e_{w_{2}-w_{4}}(b), q_{w_{1}+w_{4}}\right]} \\
\quad=-\left[e_{w_{3}-w_{1}}(a c), q_{w_{1}+w_{4}}, e_{w_{2}-w_{4}}(b), q_{w_{1}+w_{4}}\right] .
\end{gathered}
$$

Furthermore, $e_{w_{2}-w_{4}}(b)=-\left[q_{w_{1}+w_{2}}, q_{-w_{1}-w_{4}}(b)\right]$. Substituting this expression we get

$$
\begin{gathered}
\text { LHS }=\left[e_{w_{3}-w_{1}}(a c), q_{w_{1}+w_{4}}, q_{w_{1}+w_{2}}, q_{-w_{1}-w_{4}}(b), q_{w_{1}+w_{4}}\right] \\
+\left[e_{w_{3}-w_{1}}(a c), q_{w_{1}+w_{4}}, q_{-w_{1}-w_{4}}(b), q_{w_{1}+w_{2}}, q_{w_{1}+w_{4}}\right]=(I)+(I I) .
\end{gathered}
$$


Recall that we use the notation $\left[e_{w_{4}-w_{1}}(a), q_{w_{3}+w_{1}}, q_{w_{2}+w_{1}}\right]=\operatorname{Vir}(a)$. Now,

$$
\begin{aligned}
& {\left[e_{w_{3}-w_{1}}(a c), q_{w_{1}+w_{4}}, q_{w_{1}+w_{2}}\right]=-\left[e_{w_{4}-w_{1}}(a c), e_{w_{3}-w_{4}}, q_{w_{1}+w_{4}}, q_{w_{1}+w_{2}}\right]} \\
& \quad=-\left[e_{w_{4}-w_{1}}(a c),\left[e_{w_{3}-w_{4}}, q_{w_{1}+w_{4}}\right], q_{w_{1}+w_{2}}\right]
\end{aligned}
$$

since $\left[e_{w_{4}-w_{1}}(a c), q_{w_{1}+w_{4}}\right] \subseteq L_{2 w_{4}}=(0)$.

Using the fact that $\left[e_{w_{3}-w_{4}}, q_{w_{1}+w_{4}}\right]=-q_{w_{3}+w_{1}}$, our expression becomes

$$
\left[e_{w_{4}-w_{1}}(a c), q_{w_{3}+w_{1}}, q_{w_{2}+w_{1}}\right]=\operatorname{Vir}(a c) .
$$

Hence,

$$
\begin{gathered}
(I)=\left[e_{w_{3}-w_{1}}(a c), q_{w_{1}+w_{4}}, q_{w_{1}+w_{2}},\left[q_{-w_{1}-w_{4}}(b), q_{w_{1}+w_{4}}\right]\right] \\
=-\left[\operatorname{Vir}(a c), h_{w_{1}-w_{4}}(b)\right]=-h_{w_{1}-w_{4}}\left(a b^{\prime} c\right) .
\end{gathered}
$$

On the other hand,

$$
\begin{gathered}
(I I)=\left[e_{w_{3}-w_{1}}(a c), q_{w_{1}+w_{4}}, q_{-w_{1}-w_{4}}(b), q_{w_{1}+w_{2}}, q_{w_{1}+w_{4}}\right] \\
=\left[e_{w_{3}-w_{1}}(a c),\left[q_{w_{1}+w_{4}}, q_{-w_{1}-w_{4}}(b)\right], q_{w_{1}+w_{2}}, q_{w_{1}+w_{4}}\right] \\
=\left[e_{w_{3}-w_{1}}(a c), h_{w_{1}-w_{4}}(b), q_{w_{1}+w_{2}}, q_{w_{1}+w_{4}}\right]=\left[e_{w_{3}-w_{1}}(a b c), q_{w_{1}+w_{2}}, q_{w_{1}+w_{4}}\right] \\
=-\left[e_{w_{3}-w_{1}}(a b c), q_{w_{1}+w_{4}}, q_{w_{1}+w_{2}}\right]=-\operatorname{Vir}(a b c),
\end{gathered}
$$

as we have seen above. This proves the lemma.

Lemma 2.5 implies that

$$
\begin{gathered}
{\left[q_{w_{1}+w_{4}}, e_{w_{2}-w_{4}}(b)\right]\left[q_{w_{1}+w_{4}}, e_{w_{3}-w_{4}}(c)\right] e_{w_{4}-w_{1}}(a) v_{\lambda}} \\
=-\left[e_{w_{4}-w_{1}}(a),\left[q_{w_{1}+w_{4}}, e_{w_{3}-w_{4}}(c)\right],\left[q_{w_{1}+w_{4}}, e_{w_{2}-w_{4}}(b)\right]\right] v_{\lambda} \\
=\left(-h_{w_{1}-w_{4}}\left(a b^{\prime} c\right)-\operatorname{Vir}(a b c)\right) v_{\lambda} \\
=-\left\langle\lambda, h_{w_{1}-w_{4}}\right\rangle\left(a b c v^{\prime}\right)_{\lambda}+\left(\left(\mu_{0} a b c+\mu_{1} a^{\prime} b c+\mu_{2} a b^{\prime} c+\mu_{3} a b c^{\prime}\right) v\right)_{\lambda} .
\end{gathered}
$$

Here $v$ is viewed as an element from $R=F\left[t^{-1}, t\right]$, and $\mu_{0}, \mu_{1}, \mu_{2}, \mu_{3}$ are scalars from $F$.

Choosing $a=1$ we get:

$$
\begin{aligned}
& {\left[q_{w_{1}+w_{4}}, e_{w_{2}-w_{4}}(b)\right]\left[q_{w_{1}+w_{4}}, e_{w_{3}-w_{4}}(c)\right] e_{w_{4}-w_{1}} v_{\lambda} } \\
= & \left(\left(\mu_{0} b c+\mu_{2} b^{\prime} c+\mu_{3} b c^{\prime}\right) v\right)_{\lambda}-\left\langle\lambda, h_{w_{1}-w_{4}}\right\rangle\left(b c v^{\prime}\right)_{\lambda} .
\end{aligned}
$$

Hence,

$$
\begin{gathered}
{\left[q_{w_{1}+w_{4}}, e_{w_{2}-w_{4}}(b)\right]\left[q_{w_{1}+w_{4}}, e_{w_{3}-w_{4}}(c)\right] e_{w_{4}-w_{1}}(a v)_{\lambda}} \\
=\left(\left(\mu_{0} a b c+\mu_{2} a b^{\prime} c+\mu_{3} a b c^{\prime}\right) v\right)_{\lambda}-\left\langle\lambda, h_{w_{1}-w_{4}}\right\rangle\left(\left(a b c v^{\prime}\right)_{\lambda}+\left(a^{\prime} b c v\right)_{\lambda}\right) .
\end{gathered}
$$

This implies

$$
\left[q_{w_{1}+w_{4}}, e_{w_{2}-w_{4}}(b)\right]\left[q_{w_{1}+w_{4}}, e_{w_{3}-w_{4}}(c)\right]\left(e_{w_{4}-w_{1}}(a)(v)_{\lambda}-e_{w_{4}-w_{1}}(a v)_{\lambda}\right)
$$

$$
=\left(\mu_{1}+\left\langle\lambda, h_{w_{1}-w_{4}}\right\rangle\right)\left(a^{\prime} b c v\right)_{\lambda} .
$$




\section{The CASE $\left\langle\lambda, h_{w_{1}-w_{3}}\right\rangle \geq 2$}

In this section we will prove that if $\lambda$ is an integral dominant functional and $\left\langle\lambda, h_{w_{1}-w_{3}}\right\rangle \geq 2$, then for arbitrary $\beta, \alpha \in F$ the irreducible module $V(\lambda, \beta, \alpha)$ has only finitely many weights with respect to the action of $H$.

Let $\gamma=\left\langle\lambda, h_{w_{1}-w_{2}}\right\rangle\left(1-\left\langle\lambda, h_{w_{1}-w_{3}}\right\rangle\right)$ (see Lemma 2.4). Since $w_{1}-w_{2}=$ $\left(w_{1}-w_{3}\right)+\left(w_{3}-w_{2}\right)$ and the root $w_{3}-w_{2}$ is positive, we conclude that $\left\langle\lambda, h_{w_{1}-w_{2}}\right\rangle \geq$ 2 and therefore $\gamma \neq 0$. Let $\xi=\frac{\mu_{1}+\left\langle\lambda, h_{w_{1}-w_{4}}\right\rangle}{\gamma}$.

Lemma 3.1. $e_{w_{4}-w_{1}}(a) v_{\lambda}-e_{w_{4}-w_{1}}(a v)_{\lambda}-\xi q-w_{1}-w_{2} q-w_{1}-w_{3}\left(a^{\prime} v\right)_{\lambda}=0$.

Proof. Denote the left hand side of the above equality as $w$. In order to prove that $w=0$ we need only to check that $U\left(L_{+}\right) w \cap V_{\lambda}=(0)$. From the equality $(*)$ and Lemma 2.4 it follows that

$$
\left[q_{w_{1}+w_{4}}, e_{w_{2}-w_{4}}(b)\right]\left[q_{w_{1}+w_{4}}, e_{w_{3}-w_{4}}(c)\right] w=0 .
$$

Consider the element $q_{-w_{3}-w_{4}}(b) w$. We have

$$
q_{-w_{3}-w_{4}}(b) e_{w_{4}-w_{1}}(a) v_{\lambda}=q_{-w_{1}-w_{3}}(a b) v_{\lambda}=q_{-w_{1}-w_{3}}(a b v)_{\lambda}
$$

by Lemma 2.3. The last expression is also equal to $q_{-w_{3}-w_{4}}(b) e_{w_{3}-w_{1}}(a v)_{\lambda}$. Furthermore,

$$
q_{-w_{3}-w_{4}}(b) q_{-w_{1}-w_{2}} q_{-w_{1}-w_{3}} v_{\lambda}=q_{-w_{1}-w_{2}} q_{-w_{1}-w_{3}} q_{-w_{3}-w_{4}}(b) v_{\lambda}=0,
$$

since $-w_{3}-w_{4}$ is positive.

We have shown that $q_{-w_{3}-w_{4}}(R) w=(0)$.

Similarly $q_{-w_{2}-w_{4}}(R) w=(0)$.

Let us show that $e_{w_{1}-w_{4}}(R) w=(0)$. Indeed, $f\left(w_{1}-w_{4}\right)=9, f\left(-w_{1}-w_{2}\right)=$ $-2, f\left(-w_{1}-w_{3}\right)=-7$. Hence,

$$
e_{w_{1}-w_{4}}(b) q_{-w_{1}-w_{2}} q_{-w_{1}-w_{3}} v_{\lambda}=\left[e_{w_{1}-w_{4}}(b), q_{-w_{1}-w_{2}}, q_{-w_{1}-w_{3}}\right] v_{\lambda}=0 .
$$

Now

$$
\begin{gathered}
e_{w_{1}-w_{4}}(b) w=e_{w_{1}-w_{4}}(b) e_{w_{4}-w_{1}}(a) v_{\lambda}-e_{w_{1}-w_{4}}(b) e_{w_{4}-w_{1}}(a v)_{\lambda} \\
=h_{w_{1}-w_{4}}(a b) v_{\lambda}-h_{w_{1}-w_{4}}(b)(a v)_{\lambda}=0 .
\end{gathered}
$$

Since $\left[L_{w_{1}+w_{2}}, L_{w_{1}+w_{3}}\right] \subseteq e_{w_{1}-w_{4}}(R)$ it follows that for arbitrary elements $x \in$ $L_{w_{1}+w_{2}}, y \in L_{w_{1}+w_{3}}, x y w=-y x w$.

We have

$$
\begin{aligned}
& L_{w_{1}+w_{2}}=\left[q_{w_{1}+w_{4}}, e_{w_{2}-w_{4}}(R)\right]+q_{-w_{3}-w_{4}}(R), \\
& L_{w_{1}+w_{3}}=\left[q_{w_{1}+w_{4}}, e_{w_{3}-w_{4}}(R)\right]+q_{-w_{2}-w_{4}}(R) .
\end{aligned}
$$

From what we proved above, it follows that $L_{w_{1}+w_{2}} L_{w_{1}+w_{3}} w=(0)$. Together with $e_{w_{1}-w_{4}}(R) w=(0)$ it implies that $U\left(L_{+}\right) w \cap V_{\lambda}=(0)$, and therefore $w=0$. The lemma is proved.

Lemma 3.2. $e_{w_{3}-w_{1}}(a) v_{\lambda}=e_{w_{3}-w_{1}}(a v)_{\lambda}-\xi q_{-w_{1}-w_{2}} q_{-w_{1}-w_{4}}\left(a^{\prime} v\right)_{\lambda}$.

Proof. By Lemma 2.5,

$$
e_{w_{3}-w_{4}} e_{w_{4}-w_{1}}(a) v_{\lambda}=e_{w_{3}-w_{4}} e_{w_{4}-w_{1}}(a v)_{\lambda}+\xi e_{w_{3}-w_{4}} q_{-w_{1}-w_{2}} q_{-w_{1}-w_{3}}\left(a^{\prime} v\right)_{\lambda} .
$$

Since $w_{3}-w_{4}$ is positive, it implies that

$$
\left[e_{w_{3}-w_{4}}, e_{w_{4}-w_{1}}(a)\right] v_{\lambda}=\left[e_{w_{3}-w_{4}}, e_{w_{4}-w_{1}}\right](a v)_{\lambda}
$$

$+\xi q_{-w_{1}-w_{2}}\left[e_{w_{3}-w_{4}}, q_{-w_{1}-w_{3}}\right]\left(a^{\prime} v\right)_{\lambda}=e_{w_{3}-w_{1}}(a v)_{\lambda}-\xi q_{-w_{1}-w_{2}} q_{-w_{1}-w_{4}}\left(a^{\prime} v\right)_{\lambda}$.

This implies the result and proves the lemma. 
From now on in this section, unless otherwise stated, we will assume that $\left\langle\lambda, h_{w_{1}-w_{3}}\right\rangle=2$. Our first aim is to show that $e_{w_{3}-w_{1}}^{3} V_{\lambda}=(0)$.

Lemma 3.3. $e_{w_{1}-w_{3}}(a) e_{w_{3}-w_{1}}^{3} v_{\lambda}=6 \xi e_{w_{3}-w_{1}} q_{-w_{1}-w_{2}} q_{-w_{1}-w_{4}}\left(a^{\prime} v\right)$.

Proof. Taking into account that

$$
\begin{gathered}
{\left[e_{w_{1}-w_{3}}(a), e_{w_{3}-w_{1}}, e_{w_{3}-w_{1}}, e_{w_{3}-w_{1}}\right]=e_{w_{1}-w_{3}}(a) e_{w_{3}-w_{1}}^{3}} \\
-3 e_{w_{3}-w_{1}} e_{w_{1}-w_{3}}(a) e_{w_{3}-w_{1}}^{2}+3 e_{w_{3}-w_{1}}^{2} e_{w_{1}-w_{3}}(a) e_{w_{3}-w_{1}}-e_{w_{3}-w_{1}}^{3} e_{w_{1}-w_{3}}(a)=0
\end{gathered}
$$

and

$$
\begin{gathered}
e_{w_{1}-w_{3}}(a) e_{w_{3}-w_{1}}^{2}=\left[e_{w_{1}-w_{3}}(a), e_{w_{3}-w_{1}}, e_{w_{3}-w_{1}}\right] \\
+2 e_{w_{3}-w_{1}} e_{w_{1}-w_{3}}(a) e_{w_{3}-w_{1}}-e_{w_{3}-w_{1}}^{2} e_{w_{1}-w_{3}}(a) \\
=-2 e_{w_{3}-w_{1}}(a)+2 e_{w_{3}-w_{1}} e_{w_{1}-w_{3}}(a) e_{w_{3}-w_{1}}-e_{w_{3}-w_{1}}^{2} e_{w_{1}-w_{3}}(a),
\end{gathered}
$$

we get

$$
\begin{gathered}
e_{w_{1}-w_{3}}(a) e_{w_{3}-w_{1}}^{3} v_{\lambda}=3 e_{w_{3}-w_{1}}\left(-2 e_{w_{3}-w_{1}}(a)+2 e_{w_{3}-w_{1}} e_{w_{1}-w_{3}}(a) e_{w_{3}-w_{1}}\right) v_{\lambda} \\
-3 e_{w_{3}-w_{1}}^{2} e_{w_{1}-w_{3}}(a) e_{w_{3}-w_{1}} v_{\lambda}=-6 e_{w_{3}-w_{1}} e_{w_{3}-w_{1}}(a) v_{\lambda}+3 e_{w_{3}-w_{1}}^{2} h_{w_{1}-w_{3}}(a) v_{\lambda} \\
=-6 e_{w_{3}-w_{1}}\left(e_{w_{3}-w_{1}}(a v)_{\lambda}-\xi q_{-w_{1}-w_{2}} q_{-w_{1}-w_{4}}\left(a^{\prime} v\right)_{\lambda}\right)+6 e_{w_{3}-w_{1}}^{2}(a v)_{\lambda} \\
=6 \xi e_{w_{3}-w_{1}} q_{-w_{1}-w_{2}} q_{-w_{1}-w_{4}}\left(a^{\prime} v\right)_{\lambda} .
\end{gathered}
$$

The lemma is proved.

Lemma 3.4. $e_{w_{1}-w_{3}}(a) e_{w_{1}-w_{3}}(b) e_{w_{3}-w_{1}}^{3} v_{\lambda}=0$.

Proof. By Lemma 3.3, the left hand side is equal to

$$
6 \xi e_{w_{1}-w_{3}}(a) e_{w_{3}-w_{1}} q_{-w_{1}-w_{2}} q_{-w_{1}-w_{4}}\left(b^{\prime} v\right)_{\lambda} .
$$

We notice that

$$
e_{w_{1}-w_{3}}(a) q_{-w_{1}-w_{2}} q_{-w_{1}-w_{4}}\left(b^{\prime} v\right)_{\lambda}=\left[e_{w_{1}-w_{3}}(a), q_{-w_{1}-w_{2}}, q_{-w_{1}-w_{4}}\right]\left(b^{\prime} v\right)_{\lambda}=0 .
$$

Hence,

$$
\begin{gathered}
e_{w_{1}-w_{3}}(a) e_{w_{3}-w_{1}} q_{-w_{1}-w_{2}} q_{-w_{1}-w_{4}}\left(b^{\prime} v\right)_{\lambda} \\
=\left[e_{w_{1}-w_{3}}(a), e_{w_{3}-w_{1}}\right] q_{-w_{1}-w_{2}} q_{-w_{1}-w_{4}}\left(b^{\prime} v\right)_{\lambda}=h_{w_{1}-w_{3}}(a) q_{-w_{1}-w_{2}} q_{-w_{1}-w_{4}}\left(b^{\prime} v\right)_{\lambda} .
\end{gathered}
$$

From Lemma 2.3 it follows that the last expression is equal to

$$
\begin{gathered}
h_{w_{1}-w_{3}}(1) q_{-w_{1}-w_{2}} q_{-w_{1}-w_{4}}\left(a b^{\prime} v\right)_{\lambda} \\
=\left\langle\lambda+w_{3}-w_{1}, h_{w_{1}-w_{3}}(1)\right\rangle q_{-w_{1}-w_{2}} q_{-w_{1}-w_{4}}\left(a b^{\prime} v\right)_{\lambda}=0 .
\end{gathered}
$$

The lemma is proved.

\section{Lemma 3.5.}

$\left[q_{w_{1}+w_{3}}, e_{w_{4}-w_{3}}(a)\right] e_{w_{3}-w_{1}} q_{-w_{1}-w_{2}} q_{-w_{1}-w_{4}} v_{\lambda}=q_{w_{4}+w_{1}} e_{w_{3}-w_{1}} q_{-w_{1}-w_{2}} q_{-w_{1}-w_{4}}(a v)_{\lambda}$.

Proof. We have $\left[q_{w_{1}+w_{3}}, e_{w_{4}-w_{3}}(a)\right]=q_{w_{1}+w_{3}} e_{w_{4}-w_{3}}(a)-e_{w_{4}-w_{3}}(a) q_{w_{1}+w_{3}}$. Now, since the total weight of the expression $q_{w_{1}+w_{3}} e_{w_{3}-w_{1}} q_{-w_{1}-w_{2}} q_{-w_{1}-w_{4}}$ is $3 w_{3}-w_{1}$, which is positive, we only need to consider the expression

$$
q_{w_{1}+w_{3}} e_{w_{4}-w_{3}}(a) e_{w_{3}-w_{1}} q_{-w_{1}-w_{2}} q_{-w_{1}-w_{4}} v_{\lambda}=(I)+(I I),
$$

where

$$
(I)=q_{w_{1}+w_{3}} e_{w_{4}-w_{1}}(a) q_{-w_{1}-w_{2}} q_{-w_{1}-w_{4}} v_{\lambda},
$$


and

$$
(I I)=q_{w_{1}+w_{3}} e_{w_{3}-w_{1}} e_{w_{4}-w_{3}}(a) q_{-w_{1}-w_{2}} q_{-w_{1}-w_{4}} v_{\lambda} .
$$

Let us consider these expressions separately.

$$
\begin{gathered}
(I)=q_{w_{1}+w_{3}} q_{-w_{1}-w_{2}} e_{w_{4}-w_{1}}(a) q_{-w_{1}-w_{4}} v_{\lambda} \\
=\underbrace{q_{w_{1}+w_{3}} q_{-w_{1}-w_{2}} q_{-w_{1}-w_{4}} e_{w_{4}-w_{1}}(a) v_{\lambda}}_{I .1}+\underbrace{q_{w_{1}+w_{3}} q_{-w_{1}-w_{2}}\left[e_{w_{4}-w_{1}}(a), q_{-w_{1}-w_{4}}\right] v_{\lambda}}_{I .2} ; \\
(I .1)=q_{w_{1}+w_{3}} q_{-w_{1}-w_{2}} q_{-w_{1}-w_{4}} e_{w_{4}-w_{1}}(a) v_{\lambda} \\
=q_{w_{1}+w_{3}} q_{-w_{1}-w_{2}} q_{-w_{1}-w_{4}}\left(e_{w_{4}-w_{1}}(a v)_{\lambda}+\xi q_{-w_{1}-w_{2}} q_{-w_{1}-w_{3}}\left(a^{\prime} v\right)_{\lambda}\right) \\
=q_{w_{1}+w_{3}} q_{-w_{1}-w_{2}} q_{-w_{1}-w_{4}} e_{w_{4}-w_{1}}(a v)_{\lambda},
\end{gathered}
$$

because $q_{-w_{1}-w_{2}} q_{-w_{1}-w_{4}} q_{-w_{1}-w_{2}}=0$;

$$
\begin{gathered}
(I .2)=-q_{w_{1}+w_{3}} q_{-w_{1}-w_{2}} q_{-2 w_{1}}(a) v_{\lambda} \\
=q_{-w_{1}-w_{2}} q_{w_{1}+w_{3}} q_{-2 w_{1}}(a) v_{\lambda}-\left[q_{w_{1}+w_{3}}, q_{-w_{1}-w_{2}}\right] q_{-2 w_{1}}(a) v_{\lambda}=(I .2 .1)+(I .2 .2) \\
(I .2 .1)=q_{-w_{1}-w_{2}}\left[q_{w_{1}+w_{3}}, q_{-2 w_{1}}(a)\right] v_{\lambda}=-2 q_{-w_{1}-w_{2}} e_{w_{3}-w_{1}}(a) v_{\lambda} \\
=-2 q_{-w_{1}-w_{2}}\left(e_{w_{3}-w_{1}}(a v)_{\lambda}-\xi q_{-w_{1}-w_{2}} q_{-w_{1}-w_{4}}\left(a^{\prime} v\right)_{\lambda}\right)=-2 q_{-w_{1}-w_{2}} e_{w_{3}-w_{1}}(a v)_{\lambda} ; \\
(I .2 .2)=e_{w_{3}-w_{2}} q_{-2 w_{1}}(a) v_{\lambda}=q_{-2 w_{1}}(a) e_{w_{3}-w_{2}} v_{\lambda}=0
\end{gathered}
$$

since $w_{3}-w_{2}$ is positive.

$$
\begin{array}{r}
(I I)=q_{w_{1}+w_{3}} e_{w_{3}-w_{1}} e_{w_{4}-w_{3}}(a) q_{-w_{1}-w_{2}} q_{-w_{1}-w_{4}} v_{\lambda} \\
=q_{w_{1}+w_{3}} e_{w_{3}-w_{1}} q_{-w_{1}-w_{2}} e_{w_{4}-w_{3}}(a) q_{-w_{1}-w_{4}} v_{\lambda} \\
=\underbrace{q_{w_{1}+w_{3}} e_{w_{3}-w_{1}} q_{-w_{1}-w_{2}} q_{-w_{1}-w_{4}} e_{w_{4}-w_{3}}(a) v_{\lambda}}_{I I .1} \\
+\underbrace{q_{w_{1}+w_{3}} e_{w_{3}-w_{1}} q_{-w_{1}-w_{2}}\left[e_{w_{4}-w_{3}}(a), q_{-w_{1}-w_{4}}\right] v_{\lambda}}_{I I .2} .
\end{array}
$$

But

$$
\begin{gathered}
(I I .1)=q_{w_{1}+w_{3}} e_{w_{3}-w_{1}} q_{-w_{1}-w_{2}} q_{-w_{1}-w_{4}} e_{w_{4}-w_{3}}(a) v_{\lambda} \\
=q_{w_{1}+w_{3}} e_{w_{3}-w_{1}} q_{-w_{1}-w_{2}} q_{-w_{1}-w_{4}} e_{w_{4}-w_{3}}(a v)_{\lambda}
\end{gathered}
$$

by Lemma 2.3;

again by Lemma 2.3 .

$$
\begin{gathered}
(I I .2)=-q_{w_{1}+w_{3}} e_{w_{3}-w_{1}} q_{-w_{1}-w_{2}} q_{-w_{1}-w_{3}}(a) v_{\lambda} \\
=-q_{w_{1}+w_{3}} e_{w_{3}-w_{1}} q_{-w_{1}-w_{2}} q_{-w_{1}-w_{3}}(a v)_{\lambda}
\end{gathered}
$$

To summarize, we have proved that

$$
\left[q_{w_{1}+w_{3}}, e_{w_{4}-w_{3}}(a)\right] e_{w_{3}-w_{1}} q_{-w_{1}-w_{3}} q_{-w_{1}-w_{4}} v_{\lambda}=P(a v)_{\lambda},
$$

where $P$ is an operator that does not involve $a$. Choosing $a=1$, we get $P=$ $a d\left(q_{w_{4}+w_{1}}\right) a d\left(e_{w_{3}-w_{1}}\right) a d\left(q_{-w_{1}-w_{2}}\right) a d\left(q_{-w_{1}-w_{4}}\right)$. The lemma is proved.

Lemma 3.6. (i) $\left[q_{w_{1}+w_{3}}, e_{w_{4}-w_{3}}(a)\right] e_{w_{3}-w_{1}}^{3} v_{\lambda}$

$$
=-q_{w_{1}+w_{4}} e_{w_{3}-w_{1}}^{3}(a v)_{\lambda}-3 \xi e_{w_{3}-w_{1}}^{2} q_{-w_{1}-w_{2}}\left(a^{\prime} v\right)_{\lambda} .
$$

(ii) $\left[q_{w_{1}+w_{3}}, e_{w_{4}-w_{3}}(a)\right]\left[q_{w_{1}+w_{3}}, e_{w_{4}-w_{3}}(b)\right] e_{w_{3}-w_{1}}^{3} v_{\lambda}$

$$
=3 \xi q_{w_{1}+w_{4}} e_{w_{3}-w_{1}}^{2} q_{-w_{1}-w_{2}}\left(\left(a b^{\prime}-a^{\prime} b\right) v\right)_{\lambda} .
$$

(iii) $\left[q_{w_{1}+w_{3}}, e_{w_{4}-w_{3}}(a)\right]\left[q_{w_{1}+w_{3}}, e_{w_{4}-w_{3}}(b)\right]\left[q_{w_{1}+w_{3}}, e_{w_{4}-w_{3}}(c)\right] e_{w_{3}-w_{1}}^{3} v_{\lambda}=0$. 
Proof. (i) The element $q_{w_{1}+w_{3}}$ commutes with $e_{w_{3}-w_{1}}$ and $w_{1}+w_{3}$ is positive. Hence, $q_{w_{1}+w_{3}} e_{w_{3}-w_{1}}^{3} v_{\lambda}=0$.

Furthermore, $\left[e_{w_{4}-w_{3}}(a), e_{w_{3}-w_{1}}, e_{w_{3}-w_{1}}\right]=0$. Hence,

$$
\begin{gathered}
e_{w_{4}-w_{3}}(a) e_{w_{3}-w_{1}}^{3} v_{\lambda}=3 e_{w_{3}-w_{1}}^{2} e_{w_{4}-w_{3}}(a) e_{w_{3}-w_{1}} v_{\lambda}-2 e_{w_{3}-w_{1}}^{3} e_{w_{4}-w_{3}}(a) v_{\lambda} \\
=3 e_{w_{3}-w_{1}}^{2} e_{w_{4}-w_{1}}(a) v_{\lambda}+e_{w_{3}-w_{1}}^{3} e_{w_{4}-w_{3}}(a) v_{\lambda} \\
=3 e_{w_{3}-w_{1}}^{2}\left(e_{w_{4}-w_{1}}(a v)_{\lambda}+\xi q_{-w_{1}-w_{2}} q_{-w_{1}-w_{3}}\left(a^{\prime} v\right)_{\lambda}\right)+e_{w_{3}-w_{1}}^{3} e_{w_{4}-w_{3}}(a v)_{\lambda} .
\end{gathered}
$$

We have proved that

$$
\begin{gathered}
{\left[q_{w_{1}+w_{3}}, e_{w_{4}-w_{3}}(a)\right] e_{w_{3}-w_{1}}^{3} v_{\lambda}} \\
=\left[q_{w_{1}+w_{3}}, e_{w_{4}-w_{3}}\right] e_{w_{3}-w_{1}}^{3}(a v)_{\lambda}+3 \xi q_{w_{1}+w_{3}} e_{w_{3}-w_{1}}^{2} q_{-w_{1}-w_{2}} q_{-w_{1}-w_{3}}\left(a^{\prime} v\right)_{\lambda} \\
=-q_{w_{1}+w_{4}} e_{w_{3}-w_{1}}^{3}(a v)_{\lambda}+3 \xi e_{w_{3}-w_{1}}^{2} q_{w_{1}+w_{3}} q_{-w_{1}-w_{2}} q_{-w_{1}-w_{3}}\left(a^{\prime} v\right)_{\lambda} \\
=-q_{w_{1}+w_{4}} e_{w_{3}-w_{1}}^{3}(a v)_{\lambda}-3 \xi e_{w_{3}-w_{1}}^{2} e_{w_{3}-w_{2}} q_{-w_{1}-w_{3}}\left(a^{\prime} v\right)_{\lambda} \\
-3 \xi e_{w_{3}-w_{1}}^{2} q_{-w_{1}-w_{2}} q_{w_{1}+w_{3}} q_{-w_{1}-w_{3}}\left(a^{\prime} v\right)_{\lambda} \\
=-q_{w_{1}+w_{4}} e_{w_{3}-w_{1}}^{3}(a v)_{\lambda}+3 \xi e_{w_{3}-w_{1}}^{2} q_{-w_{1}-w_{2}}\left(a^{\prime} v\right)_{\lambda}-3 \xi e_{w_{3}-w_{1}}^{2} q_{-w_{1}-w_{2}} h_{w_{1}-w_{3}}\left(a^{\prime} v\right)_{\lambda} \\
=-q_{w_{1}+w_{4}} e_{w_{3}-w_{1}}^{3}(a v)_{\lambda}-3 \xi e_{w_{3}-w_{1}}^{2} q_{-w_{1}-w_{2}}\left(a^{\prime} v\right)_{\lambda} .
\end{gathered}
$$

Assertion (i) is proved.

(ii) Let us apply (i) to $\left[q_{w_{1}+w_{3}}, e_{w_{4}-w_{3}}(b)\right] e_{w_{3}-w_{1}}^{3} v_{\lambda}$ and consider both summands on the right hand side of (i) separately.

We have

$$
\begin{gathered}
{\left[q_{w_{1}+w_{3}}, e_{w_{4}-w_{3}}(a)\right] q_{w_{1}+w_{4}} e_{w_{3}-w_{1}}^{3}(b v)_{\lambda}} \\
=-q_{w_{1}+w_{4}}\left[q_{w_{1}+w_{3}}, e_{w_{4}-w_{3}}(a)\right] e_{w_{3}-w_{1}}^{3}(b v)_{\lambda} \\
=q_{w_{1}+w_{4}}\left(q_{w_{1}+w_{4}} e_{w_{3}-w_{1}}^{3}(a b v)_{\lambda}+3 \xi e_{w_{3}-w_{1}}^{2} q_{-w_{1}-w_{2}}\left(a^{\prime} b v\right)_{\lambda}\right) \\
\left.=3 \xi q_{w_{1}+w_{4}} e_{w_{3}-w_{1}}^{2} q_{-w_{1}-w_{2}}\left(a^{\prime} b v\right)_{\lambda}\right) .
\end{gathered}
$$

Acting on the second summand, we get

$$
\begin{gathered}
{\left[q_{w_{1}+w_{3}}, e_{w_{4}-w_{3}}(a)\right] e_{w_{3}-w_{1}}^{2} q_{-w_{1}-w_{2}}\left(b^{\prime} v\right)_{\lambda}} \\
=-e_{w_{3}-w_{1}}^{2}\left[q_{w_{1}+w_{3}}, e_{w_{4}-w_{3}}(a)\right] q_{-w_{1}-w_{2}}\left(b^{\prime} v\right)_{\lambda} \\
+2 e_{w_{3}-w_{1}}\left[q_{w_{1}+w_{3}}, e_{w_{4}-w_{3}}(a)\right] e_{w_{3}-w_{1}} q_{-w_{1}-w_{2}}\left(b^{\prime} v\right)_{\lambda} \\
=e_{w_{3}-w_{1}}^{2}\left[q_{w_{1}+w_{3}}, e_{w_{4}-w_{3}}(a)\right] q_{-w_{1}-w_{2}}\left(b^{\prime} v\right)_{\lambda} \\
+2 e_{w_{3}-w_{1}}\left[q_{w_{1}+w_{3}}, e_{w_{4}-w_{3}}(a), e_{w_{3}-w_{1}}\right] q_{-w_{1}-w_{2}}\left(b^{\prime} v\right)_{\lambda} \\
=e_{w_{3}-w_{1}}^{2}\left[q_{w_{1}+w_{3}}, e_{w_{4}-w_{3}}(a), q_{-w_{1}-w_{2}}\right]\left(b^{\prime} v\right)_{\lambda} \\
+2 e_{w_{3}-w_{1}}\left[q_{w_{1}+w_{3}}, e_{w_{4}-w_{1}}(a)\right] q_{-w_{1}-w_{2}}\left(b^{\prime} v\right)_{\lambda} \\
=e_{w_{3}-w_{1}}^{2} e_{w_{4}-w_{2}}(a)\left(b^{\prime} v\right)_{\lambda}+2 e_{w_{3}-w_{1}}\left[q_{w_{1}+w_{3}}, e_{w_{4}-w_{1}}(a)\right] q_{-w_{1}-w_{2}}\left(b^{\prime} v\right)_{\lambda} .
\end{gathered}
$$

The first summand of this sum is equal to $e_{w_{3}-w_{1}}^{2} e_{w_{4}-w_{2}}\left(a b^{\prime} v\right)_{\lambda}$ by Lemma 2.3. As for the second summand,

$$
\begin{gathered}
e_{w_{3}-w_{1}}\left[q_{w_{1}+w_{3}}, e_{w_{4}-w_{1}}(a)\right] q_{-w_{1}-w_{2}}\left(b^{\prime} v\right)_{\lambda} \\
=e_{w_{3}-w_{1}} q_{w_{1}+w_{3}} e_{w_{4}-w_{1}}(a) q_{-w_{1}-w_{2}}\left(b^{\prime} v\right)_{\lambda} \\
=e_{w_{3}-w_{1}} q_{w_{1}+w_{3}} q_{-w_{1}-w_{2}} e_{w_{4}-w_{1}}(a)\left(b^{\prime} v\right)_{\lambda} \\
=e_{w_{3}-w_{1}} q_{w_{1}+w_{3}} q_{-w_{1}-w_{2}} e_{w_{4}-w_{1}}\left(a b^{\prime} v\right)_{\lambda} \\
+\xi e_{w_{3}-w_{1}} q_{w_{1}+w_{3}} \underbrace{q_{-w_{1}-w_{2}} q_{-w_{1}-w_{2}}}_{0} q_{-w_{1}-w_{3}}\left(a^{\prime} b^{\prime} v\right)_{\lambda} \\
=e_{w_{3}-w_{1}} q_{w_{1}+w_{3}} q_{-w_{1}-w_{2}} e_{w_{4}-w_{1}}\left(a b^{\prime} v\right)_{\lambda} .
\end{gathered}
$$


We have shown that

$$
\left[q_{w_{1}+w_{3}}, e_{w_{4}-w_{3}}(a)\right] e_{w_{3}-w_{1}}^{2} q_{-w_{1}-w_{2}}\left(b^{\prime} v\right)_{\lambda}=P\left(a b^{\prime} v\right)_{\lambda},
$$

where $P$ is an operator which does not involve $a$ or $b$. Choosing $a=1, b=t$, we get $P=-a d\left(q_{w_{1}+w_{4}}\right) a d\left(e_{w_{3}-w_{1}}\right)^{2} a d\left(q_{-w_{1}-w_{2}}\right)$, which finishes the proof of (ii).

(iii) By using (ii) we need only to show that

$$
\left[q_{w_{1}+w_{3}}, e_{w_{4}-w_{3}}(a)\right] q_{w_{1}+w_{4}} e_{w_{3}-w_{1}}^{2} q_{-w_{1}-w_{2}} v_{\lambda}=0 .
$$

Since $\left[q_{w_{1}+w_{3}}, e_{w_{4}-w_{3}}(a)\right]$ and $q_{w_{1}+w_{4}}$ commute, the expression above is

$$
-q_{w_{1}+w_{4}}\left[q_{w_{1}+w_{3}}, e_{w_{4}-w_{3}}(a)\right] e_{w_{3}-w_{1}}^{2} q_{-w_{1}-w_{2}} v_{\lambda} .
$$

We proved above that

$$
\left[q_{w_{1}+w_{3}}, e_{w_{4}-w_{3}}(a)\right] e_{w_{3}-w_{1}}^{2} q_{-w_{1}-w_{2}} v_{\lambda}=-q_{w_{1}+w_{4}} e_{w_{3}-w_{1}}^{2} q_{-w_{1}-w_{2}}(a v)_{\lambda} .
$$

Now multiplying this expression on the left by $q_{w_{1}+w_{4}}$, we get 0 . This concludes the proof of the lemma.

Lemma 3.7. $\left[q_{w_{1}+w_{3}}, e_{w_{2}-w_{3}}(R)\right]^{3} e_{w_{3}-w_{1}}^{3} v_{\lambda}=(0)$.

Proof. Apply $a d\left(e_{w_{2}-w_{4}}\right)^{3}$ to the equality $\left[q_{w_{1}+w_{3}}, e_{w_{4}-w_{3}}(R)\right]^{3} e_{w_{3}-w_{1}}^{3} v_{\lambda}=(0)$ of Lemma 3.6(iii).

Since $\left[e_{w_{2}-w_{4}}, q_{w_{1}+w_{3}}\right]=\left[e_{w_{2}-w_{4}}, e_{w_{3}-w_{1}}\right]=\left[e_{w_{4}-w_{3}}(R), e_{w_{2}-w_{4}}, e_{w_{2}-w_{4}}\right]=(0)$, we will get

$$
\left[q_{w_{1}+w_{3}},\left[e_{w_{2}-w_{4}}, e_{w_{4}-w_{3}}(R)\right]\right]^{3} e_{w_{3}-w_{1}}^{3} v_{\lambda}=(0),
$$

completing the proof of the lemma.

Lemma 3.8. $e_{w_{3}-w_{1}}^{3} v_{\lambda}=0$.

Proof. If $e_{w_{3}-w_{1}}^{3} v_{\lambda} \neq 0$, then there exist positive roots $\alpha_{1}, \ldots, \alpha_{s}$ such that $(0) \neq$ $L_{\alpha_{1}} \cdots L_{\alpha_{s}} e_{w_{3}-w_{1}}^{3} v_{\lambda} \subseteq V_{\lambda}$. Let $s$ be the minimal number with this property. Since we can move each $L_{\alpha_{i}}$ to the right modulo shorter products, we can assume that for each $i, 1 \leq i \leq s, \alpha_{i}+w_{3}-w_{1}$ is a root or 0 and $f\left(\alpha_{i}\right) \leq f\left(w_{1}-w_{3}\right)=3$. Among all positive roots, only $w_{1}-w_{3}, w_{1}+w_{2}, w_{1}+w_{4}$ have these properties.

Suppose that

$$
(0) \neq L_{w_{1}+w_{2}}^{i} L_{w_{1}+w_{4}}^{j} e_{w_{1}-w_{3}}(R)^{k} e_{w_{3}-w_{1}}^{3} v_{\lambda} \subseteq V_{\lambda} .
$$

Then $i\left(w_{1}+w_{2}\right)+j\left(w_{1}+w_{4}\right)+(3-k)\left(w_{3}-w_{1}\right)=m\left(w_{1}+w_{2}+w_{3}+w_{4}\right)$; $0 \leq i, j, k \in \mathbf{Z}, m \in \mathbf{Z}$.

This implies $i=j=m, k=3-m \geq 0$. Hence, we have three options:

1) $k=2$ or 3 . This contradicts Lemma 3.4.

2) $k=1$. By Lemma 3.3,

$$
L_{w_{1}+w_{2}}^{2} L_{w_{1}+w_{4}}^{2} e_{w_{1}-w_{3}}(R) e_{w_{3}-w_{1}}^{3} v_{\lambda} \subseteq L_{w_{1}+w_{2}}^{2} L_{w_{1}+w_{4}}^{2} e_{w_{3}-w_{1}} q_{-w_{1}-w_{2}} q_{-w_{1}-w_{4}} v_{\lambda} .
$$

The factors in $L_{w_{1}+w_{2}}^{2} L_{w_{1}+w_{4}}^{2}$ on the right hand side anticommute, because of the minimality of $s$ and the fact that $\left[L_{w_{1}+w_{2}}, L_{w_{1}+w_{4}}\right] \subseteq e_{w_{1}-w_{3}}(R)$, which leads to case 2).

Suppose that at least one of the two $L_{w_{1}+w_{4}}$ factors lies in $q_{-w_{2}-w_{3}}(R)$. Then

$$
\begin{gathered}
\underbrace{q_{-w_{2}}(a) e_{w_{3}-w_{1}}}_{=e_{w_{3}-w_{1}} q_{-w_{2}-w_{3}}(a) q_{-w_{1}-w_{2}} q_{-w_{1}-w_{4}} v_{\lambda}+q_{-w_{2}-w_{1}}(a) q_{-w_{1}-w_{2}} q_{-w_{1}-w_{4}} v_{\lambda} .} q_{-w_{1}-w_{2}} q_{-w_{1}-w_{4}} v_{\lambda}
\end{gathered}
$$


The first summand is 0 because $-w_{2}-w_{3}$ is positive. The second summand is equal to

$$
q_{-w_{1}-w_{2}} q_{-w_{1}-w_{4}} q_{-w_{2}-w_{1}}(a) v_{\lambda}=q_{-w_{1}-w_{2}} q_{-w_{1}-w_{4}} q_{-w_{2}-w_{1}}(a v)_{\lambda}
$$

by Lemma 2.3. Now it remains to notice that $q_{-w_{1}-w_{2}} q_{-w_{1}-w_{4}} q_{-w_{1}-w_{2}}=0$.

Thus, we can assume that both factors from $L_{w_{1}+w_{4}}$ are $\left[q_{w_{1}+w_{3}}, e_{w_{4}-w_{3}}\left(a_{i}\right)\right]$, $i=1,2$.

By Lemma 3.5 we have

$$
\begin{gathered}
{\left[q_{w_{1}+w_{3}}, e_{w_{4}-w_{3}}\left(a_{1}\right)\right]\left[q_{w_{1}+w_{3}}, e_{w_{4}-w_{3}}\left(a_{2}\right)\right] e_{w_{3}-w_{1}} q_{-w_{1}-w_{2}} q_{-w_{1}-w_{4}} v_{\lambda}} \\
=\left[q_{w_{1}+w_{3}}, e_{w_{4}-w_{3}}\left(a_{1}\right)\right] q_{w_{4}+w_{1}} e_{w_{3}-w_{1}} q_{-w_{1}-w_{2}} q_{-w_{1}-w_{4}}\left(a_{2} v\right)_{\lambda} .
\end{gathered}
$$

The element $\left[q_{w_{1}+w_{3}}, e_{w_{4}-w_{3}}\left(a_{1}\right)\right]$ anticommutes with $q_{w_{4}+w_{1}}$. Hence again by Lemma 3.5,

$$
\begin{gathered}
{\left[q_{w_{1}+w_{3}}, e_{w_{4}-w_{3}}\left(a_{1}\right)\right] q_{w_{4}+w_{1}} e_{w_{3}-w_{1}} q_{-w_{1}-w_{2}} q_{-w_{1}-w_{4}}\left(a_{2} v\right)_{\lambda}} \\
=-q_{w_{4}+w_{1}} q_{w_{4}+w_{1}} e_{w_{3}-w_{1}} q_{-w_{1}-w_{2}} q_{-w_{1}-w_{4}}\left(a_{1} a_{2} v\right)_{\lambda}=0 .
\end{gathered}
$$

3) $k=0$. We have to examine $L_{w_{1}+w_{2}}^{3} L_{w_{1}+w_{4}}^{3} e_{w_{3}-w_{1}}^{3} v_{\lambda}$. As above, we conclude that factors from $L_{w_{1}+w_{2}}$ and $L_{w_{1}+w_{4}}$ anticommute modulo the previous cases $(k \geq 1)$.

From $q_{-w_{3}-w_{4}}(R)^{2} e_{w_{3}-w_{1}}^{3} v_{\lambda}=(0)$, it follows that no more than one factor from $L_{w_{1}+w_{4}}$ lies in $q_{-w_{2}-w_{3}}(R)$.

On the other hand, Lemma 3.6 (iii) implies that exactly one factor from $L_{w_{1}+w_{4}}$ lies in $q_{-w_{2}-w_{3}}(R)$. Similarly, $q_{-w_{3}-w_{4}}(R)^{2} e_{w_{3}-w_{1}}^{3} v_{\lambda}=(0)$ and Lemma 3.7 imply that exactly one factor from $L_{w_{1}+w_{2}}$ lies in $q_{-w_{3}-w_{4}}(R)$.

Now we need to show that

$$
\begin{gathered}
{\left[q_{w_{1}+w_{3}}, e_{w_{2}-w_{3}}\left(a_{1}\right)\right]\left[q_{w_{1}+w_{3}}, e_{w_{2}-w_{3}}\left(a_{2}\right)\right]\left[q_{w_{1}+w_{3}}, e_{w_{4}-w_{3}}\left(b_{1}\right)\right]\left[q_{w_{1}+w_{3}}, e_{w_{4}-w_{3}}\left(b_{2}\right)\right]} \\
q_{-w_{3}-w_{4}}\left(c_{1}\right) q_{-w_{2}-w_{3}}\left(c_{2}\right) e_{w_{3}-w_{1}}^{3} v_{\lambda}=0 .
\end{gathered}
$$

First notice that

$$
\begin{gathered}
q_{-w_{2}-w_{3}}\left(c_{2}\right) e_{w_{3}-w_{1}}^{3} v_{\lambda}=3 e_{w_{3}-w_{1}}^{2}\left[q_{-w_{2}-w_{3}}\left(c_{2}\right), e_{w_{3}-w_{1}}\right] v_{\lambda} \\
=3 e_{w_{3}-w_{1}}^{2} q_{-w_{1}-w_{2}}\left(c_{2}\right) v_{\lambda}=3 e_{w_{3}-w_{1}}^{2} q_{-w_{1}-w_{2}}\left(c_{2} v\right)_{\lambda}
\end{gathered}
$$

by Lemma 2.3. Hence, without loss of generality, we can assume that $c_{2}=1$ and, similarly, $c_{1}=1$. Moreover,

$$
\begin{gathered}
q_{-w_{3}-w_{4}} q_{-w_{2}-w_{3}} e_{w_{3}-w_{1}}^{3} v_{\lambda} \\
=6 e_{w_{3}-w_{1}}\left[q_{-w_{3}-w_{4}}, e_{w_{3}-w_{1}}\right]\left[q_{-w_{2}-w_{3}}, e_{w_{3}-w_{1}}\right] v_{\lambda}=6 e_{w_{3}-w_{1}} q_{-w_{1}-w_{4}} q_{-w_{1}-w_{2}} v_{\lambda} .
\end{gathered}
$$

Now,

$$
\left[q_{w_{1}+w_{3}}, e_{w_{4}-w_{3}}\left(b_{1}\right)\right]\left[q_{w_{1}+w_{3}}, e_{w_{4}-w_{3}}\left(b_{2}\right)\right] e_{w_{3}-w_{1}} q_{-w_{1}-w_{4}} q_{-w_{1}-w_{2}} v_{\lambda}=0
$$

follows from Lemma 3.5. This concludes the proof of the lemma.

Lemma 3.9. 1) $\left(e_{w_{4}-w_{3}}\right)^{\left\langle\lambda, h_{w_{3}-w_{4}}\right\rangle+1} v_{\lambda}=0$,

2) $\left(e_{w_{4}-w_{2}}\right)^{\left\langle\lambda, h_{w_{2}-w_{4}}\right\rangle+1} v_{\lambda}=0$. 
Proof. The only positive roots $\alpha$ such that $\alpha+w_{4}-w_{3}$ is a root and $f(\alpha) \leq$ $f\left(w_{3}-w_{4}\right)=6$ are $w_{3}-w_{4}, w_{1}+w_{2}, w_{3}-w_{2}, w_{2}-w_{4}$. Suppose that

$$
(0) \neq L_{w_{1}+w_{2}}^{i} L_{w_{3}-w_{2}}^{j} L_{w_{2}-w_{4}}^{k} L_{w_{3}-w_{4}}^{l} e_{w_{4}-w_{3}}^{q} v_{\lambda} \subseteq V_{\lambda}, \quad q=\left\langle\lambda, h_{w_{3}-w_{4}}\right\rangle+1 .
$$

Then $i\left(w_{1}+w_{2}\right)+j\left(w_{3}-w_{2}\right)+k\left(w_{2}-w_{4}\right)+(q-l)\left(w_{4}-w_{3}\right)=m\left(w_{1}+w_{2}+w_{3}+w_{4}\right)$, where $i, j, k, l, m \in \mathbf{Z}$.

This implies $i=m, i-j+k=m, j-(q-l)=m,-k+(q-l)=m$. Hence $i=m=0, j=k=q-l \geq 0$.

Now we have to examine the expression $L_{w_{3}-w_{2}}^{j} L_{w_{2}-w_{4}}^{j} L_{w_{3}-w_{4}}^{l} e_{w_{4}-w_{3}}^{q} v_{\lambda}$, where $l=q-j$.

Suppose that $l \geq 1$. There exist rational numbers $\mu, \nu$ such that for an arbitrary element $a \in R$,

$$
\begin{gathered}
e_{w_{3}-w_{4}}(a) e_{w_{4}-w_{3}}^{q} v_{\lambda}=\mu e_{w_{4}-w_{3}}^{q-2} e_{w_{4}-w_{3}}(a) v_{\lambda}+\nu e_{w_{4}-w_{3}}^{q-1} h_{w_{3}-w_{4}} v_{\lambda} \\
=\mu e_{w_{4}-w_{3}}^{q-2} e_{w_{4}-w_{3}}(a v)_{\lambda}+\nu e_{w_{4}-w_{3}}^{q-1}\left\langle\lambda, h_{w_{3}-w_{4}}\right\rangle(a v)_{\lambda}
\end{gathered}
$$

by Lemma 2.3 .

This implies that

$$
e_{w_{3}-w_{4}}(a) e_{w_{4}-w_{3}}^{q} v_{\lambda}=e_{w_{3}-w_{4}} e_{w_{4}-w_{3}}^{q}(a v)_{\lambda}=0
$$

since $q=\left\langle\lambda, h_{w_{3}-w_{4}}\right\rangle+1$.

Now let $l=0, j=q$. As above,

$$
e_{w_{2}-w_{4}}(a) e_{w_{4}-w_{3}}^{q} v_{\lambda}=q e_{w_{4}-w_{3}}^{q-1} e_{w_{2}-w_{3}}(a) v_{\lambda}=q e_{w_{4}-w_{3}}^{q-1} e_{w_{2}-w_{3}}(a v)_{\lambda} .
$$

This implies that

$$
L_{w_{3}-w_{2}}^{q} L_{w_{2}-w_{4}}^{q} e_{w_{4}-w_{3}}^{q} V_{\lambda}=L_{w_{3}-w_{2}}^{q} e_{w_{2}-w_{4}}^{q} e_{w_{4}-w_{3}}^{q} V_{\lambda},
$$

and, similarly, this expression is equal to $e_{w_{3}-w_{2}}^{q} e_{w_{2}-w_{4}}^{q} e_{w_{4}-w_{3}}^{q} V_{\lambda}$.

We have shown above that

$$
e_{w_{2}-w_{4}}^{q} e_{w_{4}-w_{3}}^{q} v_{\lambda}=q ! e_{w_{2}-w_{3}}^{q} v_{\lambda} .
$$

Now $e_{w_{3}-w_{2}}^{q} e_{w_{2}-w_{3}}^{q} v_{\lambda}=0$ because $q=\left\langle\lambda, h_{w_{3}-w_{4}}\right\rangle+1 \geq\left\langle\lambda, h_{w_{3}-w_{2}}\right\rangle+1$. This proves 1).

Let us now prove assertion 2). The only positive roots $\alpha$ such that $\alpha+w_{4}-w_{2}$ is a root and $f(\alpha) \leq f\left(w_{2}-w_{4}\right)=1$ are $w_{2}-w_{4}$ and $w_{1}+w_{4}$.

If

$$
(0) \neq L_{w_{1}+w_{4}}^{i} L_{w_{2}-w_{4}}^{j} e_{w_{4}-w_{2}}^{p} v_{\lambda} \subseteq V_{\lambda}, p=\left\langle\lambda, h_{w_{2}-w_{4}}\right\rangle+1,
$$

then $i\left(w_{1}+w_{4}\right)+(p-j)\left(w_{4}-w_{2}\right)=m\left(w_{1}+w_{2}+w_{3}+w_{4}\right)$, which implies $i=m=0$, $j=p$.

Arguing as above, we see that $L_{w_{2}-w_{4}}^{p} e_{w_{4}-w_{2}}^{p} V_{\lambda}=e_{w_{2}-w_{4}}^{p} e_{w_{4}-w_{2}}^{p} V_{\lambda}=0$.

This completes the proof of the lemma.

Lemma 3.10. Let $M \subseteq L$ be a subspace such that $M^{n} v_{\lambda}=(0)$, where $v_{\lambda} \in V_{\lambda}$. Let $1 \leq i \neq j \leq 4$ and $\bar{e}_{w_{i}-w_{j}}^{m} v_{\lambda}=0$. Suppose further that $\left[e_{w_{i}-w_{j}}, M, M\right]=(0)$. Then $\left[M, e_{w_{i}-w_{j}}\right]^{m+n} v_{\lambda}=(0)$.

Proof. From $\left[M, e_{w_{i}-w_{j}}, e_{w_{i}-w_{j}}\right]=(0)$ it follows that

$$
\left[M, e_{w_{i}-w_{j}}\right]^{m+n}=[\underbrace{M \cdots M}_{m+n}, \underbrace{e_{w_{i}-w_{j}}, \ldots, e_{w_{i}-w_{j}}}_{m+n}],
$$


where products on the left hand side and in $M \cdots M$ are taken in the associative algebra $\operatorname{End}_{F}(V)$. Hence,

$$
\left[M, e_{w_{i}-w_{j}}\right]^{m+n} v_{\lambda} \subseteq \sum_{s+r=m+n} e_{w_{i}-w_{j}}^{s} \underbrace{M \cdots M} e_{w_{i}-w_{j}}^{r} v_{\lambda} .
$$

In each nonzero summand on the right hand side $r \leq m-1$.

From $\left.\left.\left.[\underbrace{M,[M,[M, \ldots[M}_{r+1}, e_{w_{i}-w_{j}}^{r}]\right] \cdots\right]\right]=(0)$ it follows that

$$
\underbrace{M \cdots M}_{m+n} e_{w_{i}-w_{j}}^{r} \subseteq \sum_{p+q=m+n, p<m} M^{p} e_{w_{i}-w_{j}}^{r} M^{q},
$$

which implies that $q \geq n$, and therefore $M^{q} v_{\lambda}=(0)$.

Lemma 3.11. There exists $m \geq 1$ such that $e_{w_{i}-w_{j}}^{m} V_{\lambda}=(0)$ for any $1 \leq i \neq j \leq m$.

Proof. By Lemmas 3.8 and 3.9 the elements $e_{ \pm\left(w_{1}-w_{3}\right)}, e_{ \pm\left(w_{3}-w_{4}\right)}, e_{ \pm\left(w_{2}-w_{4}\right)}$ act nilpotently on $V_{\lambda}$. Now it remains to notice that those elements generate $\operatorname{sl}(4)$ and to use Lemma 3.10.

Lemma 3.12. For an arbitrary root $\alpha$ the subspace $L_{\alpha}$ acts nilpotently on $V_{\lambda}$.

Proof. Let $\alpha=w_{i}-w_{j}, 1 \leq i \neq j \leq 4, w_{i}-w_{j}$ negative. We have shown that $e_{w_{i}-w_{j}}^{m} V_{\lambda}=(0)$.

Now,

and

$$
L_{w_{i}-w_{j}}=\left[e_{w_{j}-w_{i}}(R), e_{w_{i}-w_{j}}, e_{w_{i}-w_{j}}\right]
$$

This implies that

$$
\left[e_{w_{j}-w_{i}}(R), e_{w_{i}-w_{j}}, e_{w_{i}-w_{j}}, e_{w_{i}-w_{j}}\right]=(0) .
$$

$$
L_{w_{i}-w_{j}}^{m}=[L_{w_{j}-w_{i}}^{m}, \underbrace{e_{w_{i}-w_{j}}, \ldots, e_{w_{i}-w_{j}}}_{2 m}] \subseteq \sum_{p+q=2 m} e_{w_{i}-w_{j}}^{p} L_{w_{j}-w_{i}}^{m} e_{w_{i}-w_{j}}^{q} .
$$

If $q \geq m$, then $e_{w_{i}-w_{j}}^{q} V_{\lambda}=(0)$. If $q \leq m-1$, then $f\left(m\left(w_{j}-w_{i}\right)+q\left(w_{i}-w_{j}\right)\right)>0$, and again $L_{w_{j}-w_{i}}^{m} e_{w_{i}-w_{j}}^{q} V_{\lambda}=(0)$. We have shown that $L_{w_{j}-w_{i}}^{m} V_{\lambda}=(0)$.

Let $\alpha$ be an odd root such that $L_{\alpha}$ acts on $V_{\alpha}$ nilpotently; $\alpha$ is not of the form $-2 w_{k}$. Then for arbitrary $1 \leq i \neq j \leq 4$ the subspace $\left[L_{\alpha}, e_{w_{i}-w_{j}}\right]$ acts on $V_{\lambda}$ nilpotently. Indeed, since $\alpha \neq-2 w_{i}$, we have $\left[L_{\alpha}, e_{w_{i}-w_{j}}, e_{w_{i}-w_{j}}\right]=\left[e_{w_{i}-e_{j}}, L_{\alpha}, L_{\alpha}\right]=$ (0). Now the claim follows from Lemma 3.10.

Consider a root space $L_{w_{i}+w_{j}}, 1 \leq i \neq j \leq 4$. If one of $i, j$ is equal to 1 , then $w_{i}+w_{j}>0$. Let $i \neq 1, j \neq 1$. Then $\left[L_{w_{i}+w_{j}}, e_{w_{i}-w_{1}}\right]=(0)$, but $\left\langle w_{i}+w_{j}, h_{w_{i}-w_{1}}\right\rangle \neq$ 0 . Hence, $L_{w_{i}+w_{j}}=\left[\left[e_{w_{i}-w_{1}}, e_{w_{1}-w_{i}}\right], L_{w_{i}+w_{j}}\right] \subseteq\left[e_{w_{i}-w_{1}}, L_{w_{1}+w_{j}}\right]$.

From what we proved above it follows that $L_{w_{i}+w_{j}}$ acts on $V_{\lambda}$ nilpotently.

Next, $L_{-2 w_{i}}=\left[\left[e_{w_{j}-w_{i}}, e_{w_{i}-w_{j}}\right], L_{-2 w_{i}}\right] \subseteq\left[e_{w_{j}-w_{i}}, L_{-w_{i}-w_{j}}\right]$, which implies that $L_{-2 w_{i}}$ acts on $V_{\lambda}$ nilpotently. This completes the proof of the lemma.

\section{Tensor product of modules $V(\lambda, \beta, \alpha)$}

In this section we will discuss a realization of modules $V(\beta, \alpha)$ and define a tensor product in this class.

Let $R$ be an arbitrary commutative $F$-algebra with a derivation $d: R \rightarrow R$. Recall that the Weyl algebra $W$ is $W=\sum_{i=0}^{\infty} R d^{i}, d a=a d+d(a)$. For an arbitrary scalar $\beta \in F$ consider the vector space $W_{\beta}(R, d)=\left\{a_{0} d^{\beta}+a_{1} d^{\beta-1}+\right.$ 
$\left.a_{2} d^{\beta-2}+\cdots, a_{i} \in R\right\}$, the (infinite) sums are understood formally, $\tilde{W}(R, d)=$ $\sum_{\beta \in F} W_{\beta}(R, d)$. The rule $d^{\gamma} a=\sum_{i=0}^{\infty}\left(\begin{array}{c}\gamma \\ i\end{array}\right) d^{i}(a) d^{\gamma-i}$, where $d^{i}(a)$ is the $i$-th derivative of the element $a$, makes $\tilde{W}(R, d)$ into an associative algebra, $W \subseteq \tilde{W}(R, d)$. Moreover, for each $\beta \in F$ we have $\left[R d, W_{\beta}(R, d)\right] \subseteq W_{\beta}(R, d)$. Hence $W_{\beta}(R, d)$ is a module over the Virasoro algebra $R d$.

Now consider the associative commutative algebra $\tilde{R}=R+R v, v^{2}=0$. Extend the derivation $d$ via $d(v)=-\alpha v, \alpha \in F$. Then the subspace $W_{\beta}(R, v, d)=$ $\sum_{i=0}^{\infty} R v d^{\beta-i} \subset W_{\beta}(\tilde{R}, d)$ is an $R d$-submodule of $W_{\beta}(\tilde{R}, d)$.

The following proposition is straightforward.

Proposition 4.1. $W_{\beta}(R, v, d) / W_{\beta-1}(R, v, d) \simeq V(\beta, \alpha)$.

The tensor product

$$
V\left(\beta_{1}, \alpha_{1}\right) \otimes_{F} V\left(\beta_{2}, \alpha_{2}\right)
$$

can be identified with $W_{\beta_{1}+\beta_{2}}\left(R, v_{1} v_{2}, d\right) / W_{\beta_{1}+\beta_{2}-1}\left(R, v_{1} v_{2}, d\right)$, where $R=$ $F\left[t_{1}^{-1}, t_{1}, t_{2}^{-1} t_{2}\right], d=-\partial / \partial t_{1}-\partial / \partial t_{2}$.

Since $d\left(t_{1}-t_{2}\right)=0$ it follows that $\left(t_{1}-t_{2}\right)\left(V\left(\beta_{1}, \alpha_{1}\right) \otimes V\left(\beta_{2}, \alpha_{2}\right)\right)$ is a submodule of $V\left(\beta_{1}, \alpha_{1}\right) \otimes V\left(\beta_{2}, \alpha_{2}\right)$.

Clearly, $V\left(\beta_{1}, \alpha_{1}\right) \otimes V\left(\beta_{2}, \alpha_{2}\right) /\left(t_{1}-t_{2}\right) \simeq V\left(\beta_{1}+\beta_{2}, \alpha_{1}+\alpha_{2}\right)$.

Proposition 4.2. If $V\left(\lambda_{i}, \beta_{i}, \alpha_{i}\right), i=1,2$, are conformal modules of finite type, then so is $V\left(\lambda_{1}+\lambda_{2}, \beta_{1}+\beta_{2}, \alpha_{1}+\alpha_{2}\right)$.

Proof. The $L$-modules $V\left(\lambda_{i}, \beta_{i}, \alpha_{i}\right)$ have finitely many weight spaces with respect to the Cartan subalgebra $H$ of $L$.

The tensor product $V=V\left(\lambda_{1}, \beta_{1}, \alpha_{1}\right) \otimes V\left(\lambda_{2}, \beta_{2}, \alpha_{2}\right)$ also has finitely many weight spaces.

The subspace of $V$ of weight $\lambda_{1}+\lambda_{2}$ can be identified with $V\left(\beta_{1}, \alpha_{1}\right) \otimes V\left(\beta_{2}, \alpha_{2}\right)$.

Let $M$ be the submodule of $V$ generated by $\left(t_{1}-t_{2}\right)\left(V\left(\beta_{1}, \alpha_{1}\right) \otimes V\left(\beta_{2}, \alpha_{2}\right)\right)$.

Then

$$
(V / M)_{\lambda_{1}+\lambda_{2}} \simeq V\left(\beta_{1}+\beta_{2}, \alpha_{1}+\alpha_{2}\right) .
$$

The $L$-module $V\left(\lambda_{1}+\lambda_{2}, \beta_{1}+\beta_{2}, \alpha_{1}+\alpha_{2}\right)$ is a homomorphic image of the submodule of $V / M$ generated by $(V / M)_{\lambda_{1}+\lambda_{2}}$.

Hence $V\left(\lambda_{1}+\lambda_{2}, \beta_{1}+\beta_{2}, \alpha_{1}+\alpha_{2}\right)$ has finitely many weight spaces with respect to $H$. This concludes the proof of the proposition.

Consider a copy of the algebra of Laurent polynomials $\overline{F\left[t, t^{-1}\right]}$ and make it a $W$-module via $a \bar{b}=\overline{a b}, d \bar{b}=-\bar{b}^{\prime}, a, b \in F\left[t^{-1}, t\right]$. Then the space of 8-columns ${\overline{F\left[t, t^{-1}\right]}}^{8}$ becomes a left module over $M_{8}(W)$, hence a $C K(6)$-module.

It is easy to see that this $C K(6)$-module is irreducible.

If we define the form $\left(w_{i} / w_{j}\right)=\delta_{i j}$ on $\sum_{i=1}^{4} F w_{i}$ and view functionals on $H$ as elements of $\sum_{i=1}^{4} F w_{i}$, then the highest weight of the module ${\overline{F\left[t, t^{-1}\right]}}^{8}$ is $w_{1}$, $\left(h_{w_{i}-w_{j}} \otimes a\right)(\bar{b}, 0, \ldots, 0)^{T}=\left(w_{i}-w_{j} / w_{1}\right)(\bar{b}, 0, \ldots, 0)^{T}$.

Moreover $\operatorname{Vir}(a)(\bar{b}, 0, \ldots, 0)^{T}=\left(\overline{-a b^{\prime}-a^{\prime} b}, 0, \ldots, 0\right)^{T}$.

Hence $\overline{F\left[t, t^{-1}\right]}{ }^{8} \simeq V\left(w_{1},-1,0\right)$.

Proposition 4.3. If $\lambda$ is an integral dominant functional and $\left\langle\lambda, h_{w_{1}-w_{3}}\right\rangle \geq 2$, then for arbitrary $\beta, \alpha \in F$ the irreducible module $V(\lambda, \beta, \alpha)$ has only finitely many weights with respect to the action of $H$. 
Proof. Let $\left\langle\lambda, h_{w_{1}-w_{3}}\right\rangle=k \geq 2$. By results from Section 3 the module $V^{\prime}=$ $V\left(\lambda-(k-2) w_{1}, \beta+(k-2), \alpha\right)$ has finitely many $H$-weights. Tensoring $V^{\prime}$ with ${\overline{F\left[t, t^{-1}\right]}}^{8} \simeq V\left(w_{1},-1,0\right) k-2$ times and using Proposition 4.2, we get the result.

\section{The CASe $\left\langle\lambda, h_{w_{1}-w_{3}}\right\rangle=1$}

The aim of this section is to prove the following:

Proposition 5.1. Let $\lambda$ be an integral dominant weight, such that $\left\langle\lambda, h_{w_{1}-w_{3}}\right\rangle=$ 1. Then $V(\lambda, \beta, \alpha)$ has finitely many weights with respect to $H$ if and only if $\left\langle\lambda, h_{w_{3}-w_{2}}\right\rangle=0$ and $\beta=-1$.

Suppose at first that $\lambda$ is an integral dominant weight such that $\left\langle\lambda, h_{w_{1}-w_{3}}\right\rangle=1$ and $V(\lambda, \beta, \alpha)$ has finitely many $H$-weights.

Lemma 5.2. For arbitrary elements $a \in R, v_{\lambda} \in V_{\lambda}$ we have

$$
e_{w_{3}-w_{1}}(a) v_{\lambda}=e_{w_{3}-w_{1}}(a v)_{\lambda} .
$$

Proof. Since $V(\lambda, \beta, \alpha)$ is a finite sum of eigenspaces with respect to $H$ it follows that the element $e_{w_{3}-w_{1}}$ acts on $V_{\lambda}$ nilpotently. The standard argument shows that $e_{w_{3}-w_{1}}^{2} V_{\lambda}=(0)$. Now for an arbitrary $a \in R$ we have

$$
\begin{gathered}
0=e_{w_{1}-w_{3}}(a) e_{w_{3}-w_{1}}^{2} v_{\lambda} \\
=\left[e_{w_{1}-w_{3}}(a), e_{w_{3}-w_{1}}, e_{w_{3}-w_{1}}\right] v_{\lambda}+2 e_{w_{3}-w_{1}} e_{w_{1}-w_{3}}(a) e_{w_{3}-w_{1}} v_{\lambda} \\
=-2 e_{w_{1}-w_{3}}(a) v_{\lambda}+2 e_{w_{3}-w_{1}} h_{w_{1}-w_{3}}(a) v_{\lambda}=-2\left(e_{w_{3}-w_{1}}(a) v_{\lambda}-e_{w_{1}-w_{3}}(a v)_{\lambda}\right) .
\end{gathered}
$$

This concludes the proof of the lemma.

Lemma 5.3. $\beta=-1$.

Proof.

$$
\begin{gathered}
{\left[q_{w_{1}+w_{3}}, e_{w_{2}-w_{3}}(b)\right]\left[q_{w_{1}+w_{3}}, e_{w_{4}-w_{3}}(c)\right] e_{w_{3}-w_{1}}(a) v_{\lambda}} \\
=\left[\left[q_{w_{1}+w_{3}}, e_{w_{2}-w_{3}}(b)\right],\left[\left[q_{w_{1}+w_{3}}, e_{w_{4}-w_{3}}(c)\right], e_{w_{3}-w_{1}}(a)\right]\right] v_{\lambda} \\
=\left(-h_{w_{1}-w_{3}}\left(a b^{\prime} c\right)+\operatorname{Vir}(a b c)\right) v_{\lambda}
\end{gathered}
$$

as in Lemma 2.5.

This element is equal to $\left(-a b^{\prime} c v-a b c v^{\prime}+\beta(a b c)^{\prime} v+\alpha a b c v\right)_{\lambda}$.

On the other hand, by Lemma 2.2 we have

$$
\begin{gathered}
{\left[q_{w_{1}+w_{3}}, e_{w_{2}-w_{3}}(b)\right]\left[q_{w_{1}+w_{3}}, e_{w_{4}-w_{3}}(c)\right] e_{w_{3}-w_{1}}(a) v_{\lambda}} \\
=\left[q_{w_{1}+w_{3}}, e_{w_{2}-w_{3}}(b)\right]\left[q_{w_{1}+w_{3}}, e_{w_{4}-w_{3}}(c)\right] e_{w_{3}-w_{1}}(a v)_{\lambda} \\
=\left(-h_{w_{1}-w_{3}}\left(b^{\prime} c\right)+\operatorname{Vir}(b c)\right)(a v)_{\lambda}=\left(-a b^{\prime} c v-(b c)(a v)^{\prime}+\beta(b c)^{\prime}(a v)+\alpha a b c v\right)_{\lambda} .
\end{gathered}
$$

Comparing these two expressions we see that $\beta a^{\prime} b c v=-b c a^{\prime} v$, so $\beta=-1$. This finishes the proof of the lemma.

Lemma 5.4. $\left\langle\lambda, h_{w_{3}-w_{2}}\right\rangle=0$. 
Proof. We have $q_{w_{1}+w_{2}} q_{w_{1}+w_{4}} q_{-w_{3}-w_{4}} q_{-w_{2}-w_{3}} e_{w_{3}-w_{1}}^{2} v_{\lambda}=0$. Now,

$$
q_{-w_{2}-w_{3}} e_{w_{3}-w_{1}}^{2} v_{\lambda}=2 e_{w_{3}-w_{1}}\left[q_{-w_{2}-w_{3}}, e_{w_{3}-w_{1}}\right] v_{\lambda}=2 e_{w_{3}-w_{1}} q_{-w_{1}-w_{2}} v_{\lambda} .
$$

Hence,

$$
\begin{gathered}
0=q_{w_{1}+w_{2}} q_{w_{1}+w_{4}} q_{-w_{3}-w_{4}} e_{w_{3}-w_{1}} q_{-w_{1}-w_{2}} v_{\lambda} \\
=q_{w_{1}+w_{4}} q_{-w_{3}-w_{4}} q_{w_{1}+w_{2}} e_{w_{3}-w_{1}} q_{-w_{1}-w_{2}} v_{\lambda} \\
=q_{w_{1}+w_{4}} q_{-w_{3}-w_{4}}\left[q_{w_{1}+w_{2}}, e_{w_{3}-w_{1}}\right] q_{-w_{1}-w_{2}} v_{\lambda} \\
+q_{w_{1}+w_{4}} q_{-w_{3}-w_{4}} e_{w_{3}-w_{1}}\left[q_{w_{1}+w_{2}}, q_{-w_{1}-w_{2}}\right] v_{\lambda} \\
=q_{w_{1}+w_{4}} q_{-w_{3}-w_{4}} q_{w_{2}+w_{3}} q_{-w_{1}-w_{2}} v_{\lambda}+\left\langle\lambda, h_{w_{1}-w_{2}}\right\rangle q_{w_{1}+w_{4}} q_{-w_{3}-w_{4}} e_{w_{3}-w_{1}} v_{\lambda} \\
=q_{w_{1}+w_{4}}\left[q_{-w_{3}-w_{4}}, q_{w_{2}+w_{3}}\right] q_{-w_{1}-w_{2}} v_{\lambda}-q_{w_{1}+w_{4}} q_{w_{2}+w_{3}} q_{-w_{3}-w_{4}} q_{-w_{1}-w_{2}} v_{\lambda} \\
+\left\langle\lambda, h_{w_{1}-w_{2}}\right\rangle\left[q_{w_{1}+w_{4}},\left[q_{-w_{3}-w_{4}}, e_{w_{3}-w_{1}}\right]\right] v_{\lambda} \\
=-q_{w_{1}+w_{4}} q_{-w_{1}-w_{4}} v_{\lambda}+\left\langle\lambda, h_{w_{1}-w_{2}}\right\rangle\left\langle\lambda, h_{w_{1}-w_{4}}\right\rangle v_{\lambda} \\
=\left\langle\lambda, h_{w_{1}-w_{4}}\right\rangle\left(-1+\left\langle\lambda, h_{w_{1}-w_{2}}\right\rangle\right) v_{\lambda} .
\end{gathered}
$$

Since $\left\langle\lambda, h_{w_{1}-w_{4}}\right\rangle \geq\left\langle\lambda, h_{w_{1}-w_{3}}\right\rangle=1$ it follows that $\left\langle\lambda, h_{w_{1}-w_{2}}\right\rangle=1$ and therefore $\left\langle\lambda, h_{w_{3}-w_{2}}\right\rangle=0$. This concludes the proof of the lemma.

Now we will assume that $\lambda$ is an integral dominant weight such that

$$
\left\langle\lambda, h_{w_{1}-w_{3}}\right\rangle=1,\left\langle\lambda, h_{w_{3}-w_{2}}\right\rangle=0 .
$$

Let $\beta=-1$. We will prove that $V(\lambda, \beta, \alpha)$ is a finite sum of eigenspaces with respect to $H$.

Lemma 5.5. Under the assumptions above, $e_{w_{3}-w_{1}}(a) v_{\lambda}=e_{w_{3}-w_{1}}(a v)_{\lambda}$ for arbitrary $a \in R, v_{\lambda} \in V_{\lambda}$.

Proof. The computations of Lemma 5.4 show that for $\left\langle\lambda, h_{w_{1}-w_{3}}\right\rangle=1, \beta=-1$ we have

$$
\left[q_{w_{1}+w_{3}}, e_{w_{2}-w_{3}}(R)\right]\left[q_{w_{1}+w_{3}}, e_{w_{4}-w_{3}}(R)\right]\left(e_{w_{3}-w_{1}}(a) v_{\lambda}-e_{w_{3}-w_{1}}(a v)\right)_{\lambda}=0 .
$$

Also, $q_{-w_{3}-w_{4}}(R)\left(e_{w_{3}-w_{1}}(a) v_{\lambda}-e_{w_{3}-w_{1}}(a v)_{\lambda}\right)=q_{-w_{2}-w_{3}}(R)\left(e_{w_{3}-w_{1}}(a) v_{\lambda}-\right.$ $\left.e_{w_{3}-w_{1}}(a v)_{\lambda}\right)=(0)$ by Lemma 2.3 .

This implies that $U\left(L_{+}\right)\left(e_{w_{3}-w_{1}}(a) v_{\lambda}-e_{w_{3}-w_{1}}(a v)_{\lambda}\right)=(0)$. The lemma is proved.

Lemma 5.6. $e_{w_{4}-w_{1}}(a) v_{\lambda}=e_{w_{4}-w_{1}}(a v)_{\lambda}$ for an arbitrary $a \in R$.

Proof. Denote $w=e_{w_{4}-w_{1}}(a) v_{\lambda}-e_{w_{4}-w_{1}}(a v)_{\lambda}$. Clearly, $e_{w_{1}-w_{4}}(R) w=(0)$. Since $f\left(w_{3}-w_{4}\right)>0$, it follows that $e_{w_{3}-w_{4}}(b) w=e_{w_{3}-w_{1}}(a b) v_{\lambda}-e_{w_{3}-w_{1}}(b)(a v)_{\lambda}=0$ by Lemma 5.5 .

From $\left[q_{w_{1}+w_{4}}, e_{w_{4}-w_{1}}(R)\right]=(0)$ we conclude that $q_{w_{1}+w_{4}} w=0$. Hence, $\left[q_{w_{1}+w_{4}}, e_{w_{3}-w_{4}}(R)\right] w=(0)$. Also, $q_{-w_{2}-w_{4}}(R) w=(0)$ by Lemma 2.3 applied to the root $-w_{1}-w_{2}$. We proved that $L_{w_{1}+w_{3}} w=(0)$. If $w \neq 0$, then $U\left(L_{+}\right) w \cap V_{\lambda} \neq(0)$.

It means that there exist positive roots $\alpha_{1}, \ldots, \alpha_{s}$ such that $\alpha_{1}+\cdots+\alpha_{s}+w_{4}-$ $w_{1} \in \mathbf{Z}\left(w_{1}+w_{2}+w_{3}+w_{4}\right)$ and, moreover, $\alpha_{i}+w_{4}-w_{1}$ is a negative root or 0 for any $i$. If $\alpha_{i}$ is an even root and $\alpha_{i}+w_{4}-w_{1}$ is one of the roots of Lemma 2.3 or 0 , then $e_{\alpha_{i}}(b) w=0$ again by Lemma 2.3. By Lemma $5.5 \alpha_{i}$ is not supposed to be $w_{3}-w_{4}$ as well. This rules out all even roots except $w_{2}-w_{4}$.

Of the odd roots, we have to examine $w_{1}+w_{2}$ and $w_{1}+w_{3}$, but the latter one has been ruled out above. Hence, $i\left(w_{2}-w_{4}\right)+j\left(w_{1}+w_{2}\right)+\left(w_{4}-w_{3}\right)=$ $k\left(w_{1}+w_{2}+w_{3}+w_{4}\right), i, j, k \in \mathbf{Z}, i, j \geq 0$. This equation does not have a solution. Hence $w=0$. This finishes the proof of the lemma. 
Lemma 5.7. $e_{w_{3}-w_{1}}^{2} v_{\lambda}=0$.

Proof. For an arbitrary element $a \in R$ we have

$$
\begin{gathered}
e_{w_{1}-w_{3}}(a) e_{w_{3}-w_{1}}^{2} v_{\lambda}=\left[e_{w_{1}-w_{3}}(a), e_{w_{3}-w_{1}}, e_{w_{3}-w_{1}}\right] v_{\lambda}+2 e_{w_{3}-w_{1}} h_{w_{1}-w_{3}}(a) v_{\lambda} \\
=-2 e_{w_{3}-w_{1}}(a) v_{\lambda}+2 e_{w_{3}-w_{1}} h_{w_{1}-w_{3}}(a) v_{\lambda}=0
\end{gathered}
$$

by Lemma 5.6.

Now, as in Section 3 we see that

$$
U\left(L_{+}\right) e_{w_{3}-w_{1}}^{2} v_{\lambda} \cap V_{\lambda}=L_{w_{1}+w_{2}}^{2} L_{w_{1}+w_{4}}^{2} e_{w_{3}-w_{1}}^{2} v_{\lambda} .
$$

We have $L_{w_{1}+w_{4}}=\left[q_{w_{1}+w_{3}}, e_{w_{4}-w_{3}}(R)\right]+q_{-w_{2}-w_{3}}(R)$.

Claim 1. $\left[q_{w_{1}+w_{3}}, e_{w_{4}-w_{3}}(a)\right] e_{w_{3}-w_{1}}^{2} v_{\lambda}=\left[q_{w_{1}+w_{3}}, e_{w_{4}-w_{3}}\right] e_{w_{3}-w_{1}}^{2}(a v)_{\lambda}$ for an arbitrary $a \in R$.

Indeed, $\left[L_{w_{1}+w_{4}}, e_{w_{3}-w_{1}}, e_{w_{3}-w_{1}}\right]=(0)$ implies

$$
\begin{gathered}
{\left[q_{w_{1}+w_{3}}, e_{w_{4}-w_{3}}(a)\right] e_{w_{3}-w_{1}}^{2} v_{\lambda}=2 e_{w_{3}-w_{1}}\left[\left[q_{w_{1}+w_{3}}, e_{w_{4}-w_{3}}(a)\right], e_{w_{3}-w_{1}}\right] v_{\lambda}} \\
=2 e_{w_{3}-w_{1}} q_{w_{1}+w_{3}} e_{w_{4}-w_{1}}(a) v_{\lambda}=2 e_{w_{3}-w_{1}} q_{w_{1}+w_{3}} e_{w_{4}-w_{1}}(a v)_{\lambda}
\end{gathered}
$$

by Lemma 5.6. This proves the claim.

Claim 2. $q_{-w_{2}-w_{3}}(a) e_{w_{3}-w_{1}}^{2} v_{\lambda}=q_{-w_{2}-w_{3}} e_{w_{3}-w_{1}}^{2}(a v)_{\lambda}$.

Indeed,

$$
\begin{gathered}
q_{-w_{2}-w_{3}}(a) e_{w_{2}-w_{1}}^{2} v_{\lambda}=2 e_{w_{3}-w_{1}}\left[q_{-w_{2}-w_{3}}(a), e_{w_{3}-w_{1}}\right] v_{\lambda} \\
=2 e_{w_{3}-w_{1}} q_{-w_{1}-w_{2}}(a) v_{\lambda}=2 e_{w_{3}-w_{1}} q_{-w_{1}-w_{2}}(a v)_{\lambda}
\end{gathered}
$$

by Lemma 2.3. This proves the claim.

These claims and the similar assertions for $L_{w_{1}+w_{2}} v_{\lambda}$ show that

$$
\begin{gathered}
L_{w_{1}+w_{2}}^{2} L_{w_{1}+w_{4}}^{2} e_{w_{3}-w_{1}}^{2} V_{\lambda} \\
=\left(F q_{w_{1}+w_{2}}+F q_{-w_{3}-w_{4}}\right)^{2}\left(F q_{w_{1}+w_{4}}+F q_{-w_{2}-w_{3}}\right)^{2} e_{w_{3}-w_{1}}^{2} V_{\lambda} \\
=q_{w_{1}+w_{2}} q_{w_{1}+w_{4}} q_{-w_{3}-w_{4}} q_{-w_{2}-w_{3}} V_{\lambda} .
\end{gathered}
$$

The computations of Lemma 5.4 show that, under the assumption $\left\langle\lambda, h_{w_{2}-w_{3}}\right\rangle=$ 0 , this expression is equal to 0 . This finishes the proof of the lemma.

Lemma 5.8. $e_{w_{2}-w_{3}} v_{\lambda}=0$.

Proof. If $e_{w_{2}-w_{3}} v_{\lambda} \neq 0$, then there exist positive roots $\alpha_{1}, \ldots, \alpha_{s}$ such that $\alpha_{1}+$ $\cdots+\alpha_{s}=w_{3}-w_{2}$, for each $\alpha_{1}$ the sum $\alpha_{i}+w_{2}-w_{3}$ is a negative root or 0 and $L_{\alpha_{1}} \cdots L_{\alpha_{s}} e_{w_{2}-w_{3}} v_{\lambda} \neq(0)$.

The only positive roots with the properties above are $w_{3}-w_{2}$ and $w_{1}+w_{4}$. But

$$
e_{w_{3}-w_{2}}(a) e_{w_{2}-w_{3}} v_{\lambda}=h_{w_{3}-w_{2}}(a) v_{\lambda}=\left\langle\lambda, w_{3}-w_{2}\right\rangle(a v)_{\lambda}=0 .
$$

Hence, all $\alpha_{i}$ have to be equal to $w_{1}+w_{4}, L_{w_{1}+w_{4}}^{s} e_{w_{2}-w_{3}} v_{\lambda} \neq(0)$.

But $\left[L_{w_{1}+w_{4}},\left[L_{w_{1}+w_{4}}, e_{w_{2}-w_{3}}\right]\right]=(0)$, which leads to a contradiction and finishes the proof of the lemma.

Lemma 5.9. $e_{w_{4}-w_{3}}(a) v_{\lambda}=e_{w_{4}-w_{3}}(a v)_{\lambda}$.

Proof. We have $e_{w_{4}-w_{3}}(a)=\left[e_{w_{4}-w_{1}}(a), e_{w_{1}-w_{3}}\right]$. Hence

$$
\begin{gathered}
e_{w_{4}-w_{3}}(a)=-e_{w_{1}-w_{3}} e_{w_{4}-w_{1}}(a) v_{\lambda} \\
=-e_{w_{1}-w_{3}} e_{w_{4}-w_{1}}(a v)_{\lambda}=\left[e_{w_{4}-w_{1}}, e_{w_{1}-w_{3}}\right](a v)_{\lambda}=e_{w_{4}-w_{3}}(a v)_{\lambda}
\end{gathered}
$$

by Lemma 5.6. 
Lemma 5.10. $e_{w_{4}-w_{3}}^{\left\langle\lambda, w_{3}-w_{4}\right\rangle+1} v_{\lambda}=0$.

Proof. As in the proof of Lemma 5.8, if the assertion is not true, then there exist positive roots $\alpha_{1}, \ldots, \alpha_{s}$, such that $\alpha_{1}+\cdots+\alpha_{s}+\left(\left\langle\lambda, w_{3}-w_{4}\right\rangle+1\right)\left(w_{4}-w_{3}\right) \in$ $\mathbf{Z}\left(w_{1}+\cdots+w_{4}\right), \alpha_{i}+w_{4}-w_{3}$ is a negative root or 0 . In fact, 0 is also excluded, because

$$
e_{w_{3}-w_{4}}(a) e_{w_{4}-w_{3}}^{\left\langle\lambda, w_{3}-w_{4}\right\rangle+1} v_{\lambda}=e_{w_{3}-w_{4}} e_{w_{4}-w_{3}}^{\left\langle\lambda, w_{3}-w_{4}\right\rangle+1}(a v)_{\lambda}=0
$$

by Lemma 5.9 .

The only such positive roots are $w_{3}-w_{2}, w_{2}-w_{4}, w_{1}+w_{2},-2 w_{4}$.

Hence, there exist $i, j, k, l \in \mathbf{Z}_{\geq 0}, p \in \mathbf{Z}$, such that

$i\left(w_{3}-w_{2}\right)+j\left(w_{2}-w_{4}\right)+k\left(w_{1}+w_{2}\right)-2 l w_{4}+m\left(w_{4}-w_{3}\right)=p\left(w_{1}+w_{2}+w_{3}+w_{4}\right)$,

where $m=\left\langle\lambda, w_{3}-w_{4}\right\rangle+1$.

It means that $k=p,-i+j+k=p, i-m=p,-j-2 l=p$.

The first two equalities imply that $p=k \in \mathbf{Z}_{\geq 0}, i=j$.

Now, adding the last two equalities we get $-m-2 l=2 p$, where the left hand side is negative, whereas the right hand side is positive. This concludes the proof of the lemma.

The elements $e_{ \pm\left(w_{1}-w_{3}\right)}, e_{ \pm\left(w_{2}-w_{3}\right)}, e_{ \pm\left(w_{4}-w_{3}\right)}$ generate $\operatorname{sl}(4)$ and act on $V_{\lambda}$ nilpotently. Arguing as in the proof of Lemmas 3.11 and 3.12 we get

Lemma 5.11. (1) There exists $m \geq 1$ such that $e_{w_{i}-w_{j}}^{m} V_{\lambda}=(0)$ for any $1 \leq i \neq$ $j \leq 4$.

(2) There exist $m \geq 1$ such that $L_{\alpha}^{m} V_{\lambda}=(0)$ for an arbitrary root $\alpha$.

This implies that for an integral dominant weight $\lambda$ such that $1=\left\langle\lambda, h_{w_{1}-w_{3}}\right\rangle$, $0=\left\langle\lambda, h_{w_{3}-w_{2}}\right\rangle$, the module $V(\lambda,-1, \alpha)$ is a finite sum of weight spaces with respect to the action of $H$.

\section{THE CASE $\left\langle\lambda, h_{w_{1}-w_{3}}\right\rangle=0$}

We have

$$
\left[\left[e_{w_{4}-w_{1}}(a), q_{w_{3}+w_{1}}\right], q_{w_{2}+w_{1}}\right]=-\left[\left[e_{w_{3}-w_{1}}(a), q_{w_{1}+w_{4}}\right], q_{w_{2}+w_{1}}\right]=\operatorname{Vir}(a) .
$$

If $\left\langle\lambda, h_{w_{1}-w_{3}}\right\rangle=0$ and nevertheless $V(\lambda, \beta, \alpha)$ is of finite type, then $e_{w_{3}-w_{1}} V_{\lambda}=$ (0). This implies that

$$
e_{w_{3}-w_{1}}(a) V_{\lambda}=-\frac{1}{2}\left[e_{w_{1}-w_{3}}(a), e_{w_{3}-w_{1}}, e_{w_{3}-w_{1}}\right] V_{\lambda}=(0) .
$$

Hence

$$
q_{w_{2}+w_{1}} q_{w_{1}+w_{4}} e_{w_{3}-w_{1}}(a) V_{\lambda}=\left[q_{w_{2}+w_{1}},\left[q_{w_{1}+w_{4}}, e_{w_{3}-w_{1}}(a)\right]\right] V_{\lambda}=\operatorname{Vir}(a) V_{\lambda}=(0) .
$$

Since $H \subseteq[H \otimes R, \operatorname{Vir}(R)]$ it follows that $H V_{\lambda}=(0), \lambda=0$. Then $V$ is a onedimensional module with zero multiplication, which is not viewed as irreducible.

This concludes the proof of Theorem 2.1. 


\section{JORDAN BIMODULES}

Let $V$ be a Jordan bimodule over a unital Jordan (super)algebra $J$. Then $V$ can be represented as a direct sum $V=V_{0} \oplus V_{1 / 2} \oplus V_{1}$, where $J V_{0}=(0), V_{1 / 2}$ is a one-sided Jordan bimodule (see [11]), and $V_{1}$ is a unital Jordan bimodule.

In [11 it was shown that the universal associative enveloping algebra of $J C K(R, d)$ is $M_{4}(W(R, d))$. It means that one-sided Jordan $J C K(R, d)$-bimodules are left modules over $M_{4}(W(R, d))$ or, equivalently, 4-tuples $U^{4}$, where $U$ is a left module over $W(R, d)$.

Now suppose that $R=F\left[t^{-1}, t\right], d=\frac{d}{d t}$, and the one-sided Jordan bimodule over $J C K(6)$ is conformal. Then the left module $M$ over $W(R, d)$ is a unital conformal module. Such modules correspond to left unital $\mathbf{C}[d]$-modules. Irreducible $\mathbf{C}[d]$ modules are one-dimensional and parametrized by scalars $\alpha \in F$. Indecomposable conformal modules of finite type correspond to Jordan blocks.

Let's be more precise. Let $N$ be a left $\mathbf{C}[d]$-module. Then $N\left[t^{-1}, t\right]=\left\{\sum n_{i} t^{i}\right.$, $\left.n_{i} \in N, i \in \mathbf{Z}\right\}$ is a conformal left $W\left(F\left[t^{-1}, t\right], \frac{d}{d t}\right)$-module. It is of finite type if and only if $\operatorname{dim}_{\mathbf{C}} N<\infty$. The space of 4 -tuples $N\left[t^{-1}, t\right]^{4}$ is a left associative conformal module over $M_{4}(W)$, hence a one-sided Jordan conformal module over $J C K(6)$.

Proposition 7.1. (1) Every one-sided Jordan conformal bimodule over JCK(6) is of the type $N\left[t^{-1}, t\right]^{4}$.

(2) The module $N\left[t^{-1}, t\right]^{4}$ is irreducible if and only if $N$ is one-dimensional, $N=\mathbf{C} v, v d=\alpha v, \alpha \in F$.

(3) $N\left[t^{-1}, t\right]^{4}$ is an indecomposable module of finite type if and only if $N$ is a Jordan block.

Now let $V$ be a unital irreducible conformal Jordan bimodule of finite type over $J=J C K(6)$.

In [13, 14 it was shown that the Tits-Kantor-Koecher construction $K(V)$ is a Lie conformal module of finite type over the TKK-algebra $K(J)=C K(6)$.

In [13] we proved that: (1) the reduced module $\bar{K}(V)$ is irreducible over $K(J)$ and uniquely determines the $J$-bimodule $V ;(2)$ the action of the Cartan subalgebra $H$ on $\bar{K}(V)$ is diagonalizable, and all weights of $\bar{K}(V)$ belong to the set $\left\{ \pm w_{i} \pm w_{j}, 1 \leq\right.$ $i, j \leq 4\}$.

Let $\lambda$ be the highest weight of $\bar{K}(V)$. Since the Weyl group of $s l_{4}$ is the permutation group $P_{4}$ and $f(\lambda) \geq f(\sigma(\lambda))$ for all $\sigma \in P_{4}$, the only possibilities for $\lambda$ are: $2 w_{1}, w_{1}-w_{4}, w_{1}+w_{3},-2 w_{4}$. The last two cases are ruled out by Theorem 2.1. The modules $V\left(2 w_{1}, \beta, \alpha\right), \beta, \alpha \in F$, and $V\left(w_{1}-w_{4},-1, \alpha\right), \alpha \in F$, indeed have Jordan structures.

Proposition 7.2. Unital irreducible conformal Jordan JCK(6)-bimodules of finite type form two parametric families which correspond to $V\left(2 w_{1}, \beta, \alpha\right), \beta, \alpha \in F$, and $V\left(w_{1}-w_{4},-1, \alpha\right), \alpha \in F$.

Proof. We need to show that $V\left(2 w_{1}, \beta, \alpha\right), V\left(w_{1}-w_{4},-1, \alpha\right), \alpha, \beta \in F$, are reduced Tits-Kantor-Koecher modules of the form $\bar{K}(V)$ for some unital Jordan bimodules $V$ over $J=J C K(6)$.

As in Section 4 consider the associative commutative algebra $\tilde{R}=R+R v$, $R=\mathbf{C}\left[t^{-1}, t\right], v^{2}=0$, with the derivation $d, d(t)=-1, d(v)=\alpha v$. Consider the algebra $\tilde{W}(\tilde{R}, d)=\sum_{\beta \in F} W_{\beta}(\tilde{R}, d), W_{\beta}(R, v, d)=\sum_{i=0}^{\infty} R v d^{\beta-i}$. The Lie superalgebra $L=C K_{6}$ is embedded into $M_{8}(W)$, hence into $M_{8}(\tilde{W})$. 
Consider the subspace $\left(W_{\beta}(R, v, d)\right)_{1,5}$ of matrices having $W_{\beta}(R, v, d)$ at the intersection of the 1st row and 5 th column and 0 elsewhere. It is easy to see that $\left[L_{+},\left(W_{\beta}(R, v, d)\right)_{1,5}\right]=(0)$ and for an arbitrary element $u \in\left(W_{\beta}(R, v, d)\right)_{1,5}$, arbitrary $1 \leq i \neq j \leq 4$, we have $\left[h_{w_{i}-w_{j}}, u\right]=\left(2 w_{1} / w_{i}-w_{j}\right) u$. Let $U_{\beta}=$ $U(L)\left(W_{\beta}(R, v, d)\right)_{1,5}$ be the $L$-submodule generated by $\left(W_{\beta}(R, v, d)\right)_{1,5}$ in $M_{8}(\tilde{W})$. Clearly, $2 w_{1}$ is the highest weight of this submodule and $\left(U_{\beta}\right)_{2 w_{1}}=\left(W_{\beta}(R, v, d)\right)_{1,5}$.

The $L$-submodule $U_{\beta-1}$ of $U_{\beta}$ is generated by $\left(W_{\beta-1}(R, v, d)\right)_{1,5}, U_{\beta} / U_{\beta-1}=$ $U(L)\left(W_{\beta}(R, v, d) / W_{\beta-1}(R, v, d)\right)$, hence $U_{\beta} / U_{\beta-1}=U(L)\left(U_{\beta} / U_{\beta-1}\right)_{2 w_{1}}$, and $\left(U_{\beta} / U_{\beta-1}\right)_{2 w_{1}} \simeq V(\beta, \alpha)$.

Hence $V\left(2 w_{1}, \beta, \alpha\right)$ is a homomorphic image of the module $U_{\beta} / U_{\beta-1}$. Then all weights of $V\left(2 w_{1}, \beta, \alpha\right)$ belong to the set $\left\{ \pm w_{i} \pm w_{j} .1 \leq i, j \leq 4\right\}$, which implies that $V\left(2 w_{1}, \beta, \alpha\right) \simeq K(\bar{V})$ for some irreducible unital Jordan $J$-bimodule $V$.

Now let's turn to bimodules $V\left(2 w_{1}, \beta, \alpha\right)$. Consider the Cheng-Kac superalgebra $C K(\tilde{R}, d)$ and the subspace $e_{w_{1}-w_{4}}(R v)$. This subspace generates the $L$-submodule which is isomorphic to $V\left(w_{1}-w_{4},-1, \alpha\right)$. This concludes the proof of the proposition.

\section{ACKNOWLEDGMENTS}

The authors are grateful to the referee for numerous helpful suggestions.

\section{REFERENCES}

[1] Carina Boyallian, Victor G. Kac, and José I. Liberati, Irreducible modules over finite simple Lie conformal superalgebras of type K, J. Math. Phys. 51 (2010), no. 6, 063507, 37, DOI 10.1063/1.3397419. MR2676484 (2011j:17046)

[2] Carina Boyallian, Victor G. Kac, and José I. Liberati, Classification of Finite Irreducible Modules over the Lie Conformal Superalgebra $C_{6}$, Comm. Math. Phys. 317 (2013), no. 2, 503-546, DOI 10.1007/s00220-012-1623-8. MR.3010193

[3] Carina Boyallian, Victor G. Kac, Jose I. Liberati, and Alexei Rudakov, Representations of simple finite Lie conformal superalgebras of type $W$ and S, J. Math. Phys. 47 (2006), no. 4, 043513, 25, DOI 10.1063/1.2191788. MR2226350 (2007h:17031)

[4] Shun-Jen Cheng and Victor G. Kac, Erratum: "Conformal modules" [Asian J. Math. 1 (1997), no. 1, 181-193; MR1480993 (98j:17026)], Asian J. Math. 2 (1998), no. 1, 153-156. MR $1656556(99 \mathrm{k}: 17046)$

[5] Shun-Jen Cheng and Victor G. Kac, A new $N=6$ superconformal algebra, Comm. Math. Phys. 186 (1997), no. 1, 219-231, DOI 10.1007/BF02885679. MR1462763 (99f:17029)

[6] Davide Fattori and Victor G. Kac, Classification of finite simple Lie conformal superalgebras, J. Algebra 258 (2002), no. 1, 23-59, DOI 10.1016/S0021-8693(02)00504-5. Special issue in celebration of Claudio Procesi's 60th birthday. MR 1958896 (2004b:17017)

[7] Pavel Grozman, Dimitry Leites, and Irina Shchepochkina, Lie superalgebras of string theories, Acta Math. Vietnam. 26 (2001), no. 1, 27-63. MR1828365 (2002c:17034)

[8] V. G. Kac, Classification of simple Z-graded Lie superalgebras and simple Jordan superalgebras, Comm. Algebra 5 (1977), no. 13, 1375-1400. MR0498755 (58 \#16806)

[9] Victor Kac, Vertex algebras for beginners, 2nd ed., University Lecture Series, vol. 10, American Mathematical Society, Providence, RI, 1998. MR1651389 (99f:17033)

[10] C. Martinez and E. Zelmanov, Simple finite-dimensional Jordan superalgebras of prime characteristic, J. Algebra 236 (2001), no. 2, 575-629, DOI 10.1006/jabr.2000.8456. MR.1813492 (2002e:17042)

[11] Consuelo Martínez and Efim Zelmanov, Specializations of Jordan superalgebras, Canad. Math. Bull. 45 (2002), no. 4, 653-671, DOI 10.4153/CMB-2002-059-8. Dedicated to Robert V. Moody. MR1941232 (2003k:17040)

[12] Consuelo Martínez and Efim I. Zelmanov, Lie superalgebras graded by $P(n)$ and $Q(n)$, Proc. Natl. Acad. Sci. USA 100 (2003), no. 14, 8130-8137 (electronic), DOI 10.1073/pnas.0932706100. MR 1989345(2005c:17047) 
[13] Consuelo Martínez and Efim Zelmanov, Representation theory of Jordan superalgebras. I, Trans. Amer. Math. Soc. 362 (2010), no. 2, 815-846, DOI 10.1090/S0002-9947-09-04883-1. MR2551507 (2011a:17049)

[14] Efim Zelmanov, On the structure of conformal algebras, Combinatorial and computational algebra (Hong Kong, 1999), Contemp. Math., vol. 264, Amer. Math. Soc., Providence, RI, 2000, pp. 139-153, DOI 10.1090/conm/264/04216. MR.1800693 (2001j:17048)

Departamento de Matemáticas, Universidad de Oviedo, C/ Calvo Sotelo s/n, 33007 OVIEDO, SPAIN

E-mail address: cmartinez@uniovi.es

Department of Mathematics, University of California San Diego, la Jolla, California 92093-0112 - and - Korean Institute of Advaced Study, Seoul, South Korea - And King Abdulaziz, Jeddah, Saudi Arabia

E-mail address: ezelmano@maths.ucsd.edu 